\title{
RELAY Subgroup Analyses by EGFR Ex19del and Ex21L858R Mutations for Ramucirumab Plus Erlotinib in Metastatic Non-Small Cell Lung Cancer
}

Kazuhiko Nakagawa', Ernest Nadal ${ }^{2}$, Edward B. Garon ${ }^{3}$, Makoto Nishio ${ }^{4}$, Takashi Seto ${ }^{5}$, Nobuyuki Yamamoto ${ }^{6}$, Keunchil Park ${ }^{7}$, Jin-Yuan Shih ${ }^{8}$, Luis Paz-Ares ${ }^{9}$, Bente Frimodt-Moller ${ }^{10}$, Annamaria H. Zimmermann"1 ${ }^{11}$, Sameera Wijayawardana"1 ${ }^{11}$ Carla Visseren-Grul ${ }^{12}$, and Martin Reck ${ }^{13}$; For the RELAY study investigators

\section{ABSTRACT}

Purpose: In EGFR-mutated metastatic non-small cell lung cancer (NSCLC), outcomes from EGFR tyrosine kinase inhibitors have differed historically by mutation type present, with lower benefit reported in patients with ex21L858R versus ex19del mutations. We investigated if $E G F R$-activating mutation subtypes impact treatment outcomes in the phase III RELAY study. Associations between EGFR mutation type and preexisting co-occurring and treatmentemergent genetic alterations were also explored.

Materials and Methods: Patients with metastatic NSCLC, an EGFR ex19del or ex21L858R mutation, and no central nervous system metastases were randomized (1:1) to erlotinib (150 mg/day) with either ramucirumab (10 mg/kg; RAM+ERL) or placebo (PBO+ERL), every 2 weeks, until RECIST v1.1-defined progression or unacceptable toxicity. The primary endpoint was progressionfree survival (PFS). Secondary and exploratory endpoints included overall response rate (ORR), duration of response (DOR),

\section{Introduction}

Non-small cell lung cancer (NSCLC) is a molecularly heterogeneous disease. Approximately $40 \%$ to $60 \%$ of East Asian patients and $10 \%$ to $20 \%$ of Caucasian patients with NSCLC harbor a somatic

\begin{abstract}
'Department of Medical Oncology, Kindai University Faculty of Medicine, Osaka, Japan. ${ }^{2}$ Department of Medical Oncology, Catalan Institute of Oncology, IDIBELL, L'Hospitalet de Llobregat, Barcelona, Spain. ${ }^{3}$ David Geffen School of Medicine at University of California Los Angeles/TRIO-US Network, Los Angeles, California. ${ }^{4}$ The Cancer Institute Hospital of JFCR, Tokyo, Japan. ${ }^{5}$ Kyushu Cancer Center, Fukuoka, Japan. ${ }^{6}$ Wakayama Medical University Hospital, Wakayama, Japan. ${ }^{7}$ Samsung Medical Center, Seoul, South Korea. ${ }^{8}$ Department of Internal Medicine, National Taiwan University Hospital, Taipei City, Taiwan. ${ }^{9}$ Hospital Universitario 12 de Octubre, CNIO-H12O Lung Cancer Unit, Universidad Complutense \& CiberOnc, Madrid, Spain. ${ }^{10}$ Eli Lilly and Company, Copenhagen, Denmark. "Eli Lilly and Company, Indianapolis, Indiana. ${ }^{12}$ Lilly Oncology, Utrecht, the Netherlands. ${ }^{13}$ LungenClinic, Airway Research Center North (ARCN), German Center for Lung Research (DZL), Grosshansdorf, Germany.
\end{abstract}

Note: Supplementary data for this article are available at Clinical Cancer Research Online (http://clincancerres.aacrjournals.org/).

RELAY investigators are listed in Supplementary Material.

Corresponding Author: Kazuhiko Nakagawa, Department of Medical Oncology, Kindai University, Osaka 589-8511, Japan. E-mail: nakagawa@med.kindai.ac.jp

Clin Cancer Res 2021;XX:XX-XX

doi: 10.1158/1078-0432.CCR-21-0273

This open access article is distributed under Creative Commons AttributionNonCommercial-NoDerivatives License 4.0 International (CC BY-NC-ND).

(C)2021 The Authors; Published by the American Association for Cancer Research
PFS2, time-to-chemotherapy (TTCT), safety, and next-generation sequencing analyses.

Results: Patients with ex19del and ex21L858R mutations had similar clinical characteristics and comutational profiles. One-year PFS rates for ex19del patients were $74 \%$ for RAM+ERL versus $54 \%$ for PBO+ERL; for ex21L858R rates were 70\% (RAM+ERL) versus $47 \%(\mathrm{PBO}+\mathrm{ERL})$. Similar treatment benefits (ORR, DOR, PFS2, and TTCT) were observed in RAM+ERL-treated patients with ex19del and ex21L858R. Baseline TP53 comutation was associated with superior outcomes for RAM+ERL in both ex19del and ex21L858R subgroups. EGFR T790M mutation rate at progression was similar between treatment arms and by mutation type.

Conclusions: RAM+ERL provided significant clinical benefit for both EGFR ex19del and ex21L858R NSCLC, supporting this regimen as suitable for patients with either of these EGFR mutation types.

driver mutation in the EGFR gene (EGFRm; refs. 1,2). Approximately $90 \%$ of EGFR-activating mutations involve deletions in exon 19 (ex19del) or a substitution mutation in exon 21 , specifically Leu858Arg (ex21L858R; refs. 1, 3, 4). Although ex19del constitutes nearly 50\% and ex21L858R $40 \%$ of EGFR-activating mutations, variation in these rates is observed between regions $(1,2)$. Ex21L858R is more prevalent in Asian countries, particularly in Japan $(5,6)$.

EGFR-activating mutations are also a major predictive factor for response to small-molecule EGFR tyrosine kinase inhibitor (TKI) therapies. However, the degree of benefit may differ based on the EGFR mutation type, which has been shown to be an independent prognostic marker $(7,8)$. Indeed, although both ex19del and ex21L858R mutations benefit from EGFR-TKI treatment, subgroup analyses of several landmark studies in EGFRm NSCLC indicate that patients with ex21L858R derive more modest benefit with EGFR-TKI monotherapy than patients with ex19del, as represented by its lower median progression-free survival (PFS), smaller delta PFS, and higher PFS HR (Table 1). The difference in PFS outcomes between ex19del and ex21L858R is independent of EGFR-TKI generation used and is observed with first-, second-, and third-generation EGFR-TKIs. As treatment benefit in a given trial can be difficult to understand by analyzing results using a single measure of PFS, the totality of PFS data should be considered. Treatment benefit is most reflectively reported using a combination of various absolute (median and delta PFS, and 1-year PFS rates) and relative (HR) PFS measures, which have complementary roles in fully assessing the results of comparative time-to-event analyses and together provide the most complete insights into the different outcomes between ex19del and ex21L858R (9-11). Meta-analyses and real-world evidence studies 


\section{Translational Relevance}

Previous evidence suggests that in EGFR-mutated metastatic non-small cell lung cancer (NSCLC), patients with ex21L858R mutation have a poorer prognosis and response to treatment with EGFR-tyrosine kinase inhibitor (TKI) monotherapy than patients with ex19del mutation. Results from RELAY showed that combination of the antiangiogenic agent ramucirumab with EGFR-TKI targeted therapy provided significant and similar clinical benefit for both EGFR ex19del and ex21L858R NSCLC, suggesting that the addition of an antiangiogenic agent may abrogate the differential efficacy observed in ex21L858R patients compared with ex19del patients when treated with EGFR-TKI monotherapy. These findings may help clinicians provide a personalized treatment strategy and allow treatment decisions to be made on a more rational basis.

have provided further evidence for worse PFS outcomes and lower response rates after EGFR-TKI therapy with ex21L858R compared with ex19del (12-14). The different prognostic and predictive roles for ex19del and ex21L858R have led to the widespread acceptance of these mutations as an essential stratification factor in EGFRm NSCLC clinical trials and thereby enhanced the design and interpretation of these studies (13).

The potential to further improve patient outcomes by combining an EGFR-TKI with an antiangiogenesis agent is supported by strong preclinical and, more recently, clinical trial evidence (15-20). However, there is a paucity of published literature on how dual EGFR/VEGF pathway inhibition impacts outcomes by EGFR mutation subtypes. In the randomized phase III RELAY trial for the firstline treatment of EGFRm NSCLC, ramucirumab plus erlotinib (RAM+ERL) showed a consistent PFS treatment benefit compared with placebo plus erlotinib (PBO+ERL) among patients with ex19del or ex21L858R (19), with similar median PFS, delta PFS improvement, and HRs (Table 1), and a clear and early separation of Kaplan-Meier PFS curves for both ex19del and ex21L858R patients. With a median PFS of 19.4 months and an absolute PFS improvement of 8.2 months versus single-agent EGFR-TKI, these are among the best reported outcomes to date for a subgroup of patients with ex21L858R, albeit in a population without central nervous system (CNS) metastases.

As noted above, it is important to explore the context specificity of EGFR mutation types to different therapeutic strategies. To address this, we explored more comprehensively if the EGFRactivating mutation subtypes impact treatment outcomes in RELAY. In addition, potential associations between EGFR mutation type and baseline characteristics, patterns of progression, treatment patterns post-RELAY study treatment discontinuation, and preexisting co-occurring and treatment-emergent genetic alterations were explored.

\section{Materials and Methods}

\section{Study design and patients}

Study design and patient eligibility were detailed previously (19). Briefly, RELAY is a global, double-blind, placebo-controlled, randomized phase III study that enrolled patients with untreated metastatic NSCLC with an EGFR ex19del or ex21L858R mutation as tested locally. Random assignment was stratified according to EGFR mutation subtype (ex19del vs. ex21L858R), local EGFR testing method (therascreen/cobas vs. other PCR/sequencing-based methods), sex (male vs. female), and region (East Asia vs. other). Randomized patients (1:1) received either intravenous ramucirumab $(10 \mathrm{mg} / \mathrm{kg})$ or matching placebo every 2 weeks, with erlotinib ( $150 \mathrm{mg}$ oral) daily. Study treatment continued until radiographic progression as assessed by the investigator according to RECIST v1.1, unacceptable toxicity, withdrawal of consent, noncompliance, or investigator decision. Use of subsequent treatment was at the discretion of the investigator. The trial adhered to the Declaration of Helsinki, the International Council for Harmonisation Guideline for Good Clinical Practice, and applicable local regulations. All patients provided written informed consent. The trial is registered at ClinicalTrials.gov (identifier: NCT02411448).

\section{Assessments}

Tumor assessments were conducted using RECIST v1.1 at baseline, every 6 weeks for 72 weeks, every 12 weeks thereafter until disease progression or unacceptable toxicity, and at the 30-day follow-up visit. Adverse events (AE) were assessed at every cycle and graded according to the National Cancer Institute Common Terminology Criteria for Adverse Events (v4.0).

Confirmatory central testing for ex19del and ex21L858R was conducted on archival tissue samples collected at baseline and using the commercial therascreen assay. Central testing did not inform eligibility or study enrollment. Plasma samples were prospectively collected at baseline, Day 1 Cycle 4, and at the 30-day post-study treatment discontinuation follow-up visit, and were evaluated with Guardant360 next-generation sequencing (NGS; Guardant Health).

\section{Analysis populations and study endpoints}

Efficacy analyses were conducted in the population of randomly assigned patients. Safety analyses were evaluated in patients who received at least one dose of study drug. NGS analyses were conducted in patients with valid baseline and/or 30-day follow-up samples depending on whether baseline, treatment-emergent, or postprogression follow-up mutation profiles were being investigated.

The primary endpoint was PFS (investigator-assessed). Secondary endpoints included safety and tolerability, response rates, duration of response (DOR), and interim overall survival (iOS). Prespecified PFS2 and post hoc analyses of patterns of progression, time-to-response, time-to-chemotherapy (TTCT), and NGS biomarker analyses were exploratory (see Supplementary Materials for definitions of clinical endpoints). Other protocol-specified endpoints are not presented here (21).

\section{Statistical analyses}

Although EGFR mutation subtype was a prespecified subgroup, RELAY was not powered for analysis of this or any other subgroup. HR and $95 \%$ confidence intervals (CI) were estimated using an unstratified Cox proportional hazards model. Kaplan-Meier estimation was used to plot time-to-event data, as well as to provide summary statistics. Differences between response rates and associated 95\% CIs for treatment arms by mutation subgroup were calculated using Fisher exact method. Descriptive summary statistics were used for safety analyses. Sankey diagrams were constructed to summarize post-study treatment discontinuation therapy across both arms and to explore any treatment patterns observed. Relationships between somatic gene alterations and EGFR-activating mutation subgroup on PFS were explored using interaction tests from the Cox regression model. Fisher exact method compared mutation frequencies across treatment arms within each EGFR mutation subgroup. Additional methodologic details for the exploratory biomarker analyses are provided in the 


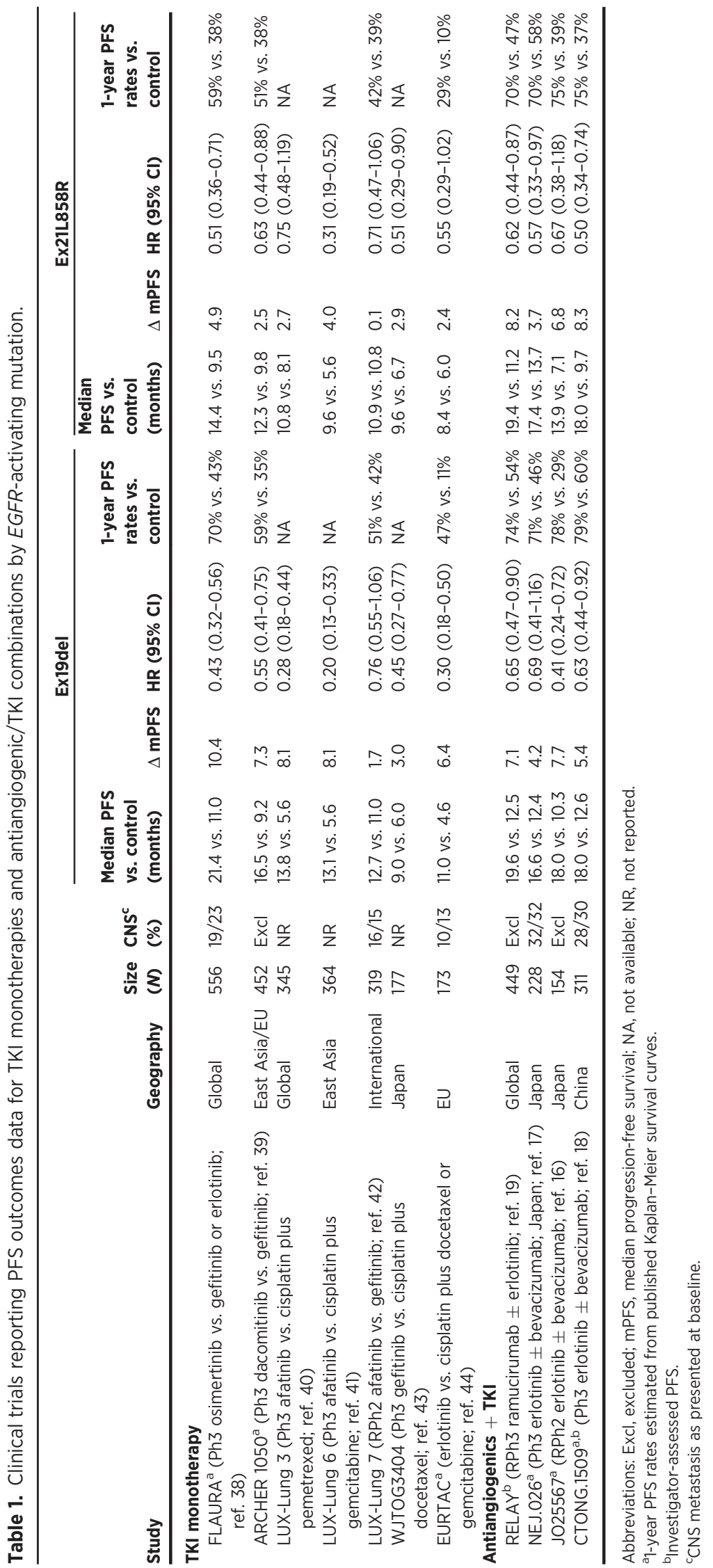


Supplementary Materials and Methods. SAS v9.4 and SAS VA v7.3 (SAS Institute) were used for all statistical analyses.

\section{Results}

\section{Patients, EGFR testing, and baseline characteristics}

In RELAY, 449 patients (intention-to-treat population) were randomized between January 28, 2016 and February 1, 2018 to either RAM+ERL or PBO+ERL treatment arms (Supplementary Fig. S1). Two patients were excluded from this analysis as they were subsequently shown not to harbor an ex19del or ex21L858R mutation. Thus, the total population for the efficacy analyses by EGFR mutation subgroups was 447 patients [243 (54\%) ex19del and 204 (46\%) ex21L858R]. The safety analyses excluded three of the 447 patients who did not receive any study drug (one ex19del and two ex21L858R patients); all were assigned to treatment with RAM+ERL. As of primary data cutoff (January 23, 2019), 63 ex19del $(59 \%$ RAM+ERL, 41\% PBO+ERL) and 44 ex21L858R (61\% RAM+ERL, $39 \% \mathrm{PBO}+\mathrm{ERL}$ ) patients remained on study treatment (Supplementary Fig. S1).

Central tissue testing results corroborated the local EGFR testing results, with $97 \%$ of centrally tested patients (305 of 316 evaluable results) having an EGFR-activating mutation detected (53\% ex19del and 44\% ex21L858R; ref. 19). In addition, 324 patients had evaluable baseline plasma NGS results, with ex19del and ex21L858R mutations present at equivalent rates (50\%; Supplementary Fig. S1). Unless otherwise indicated, subsequent analyses for this report used baseline EGFR-activating mutation status as determined by local testing.

The majority of patients in each EGFR mutation subgroup, regardless of treatment arm, were female, never smokers, had similar median age, and an Eastern Cooperative Oncology Group performance status of 0 (Table 2). Ex21L858R was more prevalent than ex19del among patients of Asian race (83\% vs. $72 \%)$ and females ( $67 \%$ vs. $60 \%$ ); bone metastases were more common in patients with ex21L858R than with ex19del (34\% vs. 26\%). Other baseline patient and disease characteristics were similar between EGFR mutation subgroups.

\section{Efficacy}

With a median follow-up of 20.7 months (range, 0.1-35.4), PFS treatment benefit with RAM+ERL was similar between EGFR mutation subgroups (Table 1; ref. 19). Improvements in median PFS among patients receiving RAM+ERL versus $\mathrm{PBO}+\mathrm{ERL}$ were 7.1 and 8.2 months for ex19del and ex21L858R subgroups, respectively. One-year PFS rates for ex19del patients were $74 \%$ (95\% CI, 64.1-80.8) for RAM+ERL versus 54\% (95\% CI, 43.9-62.2) for $\mathrm{PBO}+\mathrm{ERL}$, and for ex21L858R patients were 70\% (95\% CI, 58.9-77.9) for RAM+ERL versus 47\% (95\% CI, 37.0-57.1) for PBO+ERL (Fig. 1A; Table 1; ref. 19).

Irrespective of treatment arm, stable disease (SD) was reported more frequently in ex21L858R patients (25\%) than ex19del patients (15\%), whereas partial response (PR) was more prevalent in ex19del patients (79\%) than in ex21L858R patients (69\%). Overall response rate (ORR) was lower in ex21L858R patients (70\%) than ex19del patients (81\%), with the lowest ORR observed in the ex21L858R subgroup treated with PBO+ERL (ex19del: RAM+ERL 79\%, PBO+ERL 83\%; ex21L858R: RAM+ERL 74\%, PBO+ERL 66\%; Table 3; Supplementary Fig. S2). Disease control rate was high $(>90 \%)$ and similar in the ex19del and ex21L858R subgroups (Table 3; Supplementary Fig. S2). The median time-to-response for patients was similar (1.4-1.5 months) across mutation subgroups and treatment arms (Table 3).

DOR analysis included fewer ex21L858R patients as SD was more prevalent in this subgroup. For both mutation subgroups, DOR favored the RAM+ERL versus the PBO+ERL arm (Fig. 1B). Relative to the ex21L858R subgroup, the ex19del subgroup treated with RAM+ERL exhibited a numerically longer median DOR [18.2 (95\% CI, 13.8-20.9) vs. 16.2 (95\% CI, 12.5-20.1) months].

Table 2. Baseline patient and disease characteristics by baseline EGFR-activating mutation subgroup and by treatment arms.

\begin{tabular}{|c|c|c|c|c|c|c|c|}
\hline & & \multicolumn{2}{|c|}{ Total } & \multicolumn{2}{|c|}{ Ex19del } & \multicolumn{2}{|c|}{ Ex21L858R } \\
\hline & & $\begin{array}{l}\text { Ex19del } \\
(N=243)\end{array}$ & $\begin{array}{l}\text { Ex21L858R } \\
(N=204)\end{array}$ & $\begin{array}{l}\text { RAM+ERL } \\
(N=123)\end{array}$ & $\begin{array}{l}\text { PBO+ERL } \\
(N=120)\end{array}$ & $\begin{array}{l}\text { RAM+ERL } \\
(N=99)\end{array}$ & $\begin{array}{l}\text { PBO+ERL } \\
(N=105)\end{array}$ \\
\hline Sex, $n(\%)$ & Female & $146(60)$ & $136(67)$ & $74(60)$ & $72(60)$ & $66(67)$ & $70(67)$ \\
\hline Age in years, median (range) & & $63(23-84)$ & $66(41-89)$ & $64(27-84)$ & $63(23-83)$ & $67(44-86)$ & $66(41-89)$ \\
\hline \multirow[t]{2}{*}{ Race $^{a}$} & Asian & $174(72)$ & $170(83)$ & $89(72)$ & $85(71)$ & $81(82)$ & $89(85)$ \\
\hline & Caucasian & $67(28)$ & $33(16)$ & $34(28)$ & $33(28)$ & $18(18)$ & $15(14)$ \\
\hline Smoking history, $n(\%)$ & Never & $144(59)$ & $127(62)$ & $71(58)$ & $73(61)$ & $61(62)$ & $66(63)$ \\
\hline ECOG performance status, $n$ (\%) & 0 & $131(54)$ & $103(51)$ & $65(53)$ & $66(55)$ & $50(51)$ & $53(51)$ \\
\hline \multirow[t]{2}{*}{ Disease classification, $n$ (\%) } & Primary metastatic & $207(85)$ & $178(87)$ & $103(84)$ & $104(87)$ & $91(92)$ & $87(83)$ \\
\hline & Recurrent metastatic & $36(15)$ & $26(13)$ & $20(16)$ & $16(13)$ & $8(8)$ & $18(17)$ \\
\hline \multirow[t]{5}{*}{ Metastases sites, $n(\%)$} & Lung & $224(92)$ & $188(92)$ & $112(91)$ & $112(93)$ & $93(94)$ & $95(91)$ \\
\hline & Lymph & $152(63)$ & $127(62)$ & $78(63)$ & $74(62)$ & $64(65)$ & $63(60)$ \\
\hline & Bone & $63(26)$ & $69(34)$ & $35(29)$ & $28(23)$ & $33(33)$ & $36(34)$ \\
\hline & Liver & $26(11)$ & $19(9)$ & $11(9)$ & $15(13)$ & $10(10)$ & $9(9)$ \\
\hline & Other & $146(60)$ & $104(51)$ & $70(57)$ & $76(63)$ & $48(49)$ & $56(53)$ \\
\hline \multirow[t]{5}{*}{ Number of metastatic sites } & 1 & $29(12)$ & $23(11)$ & $12(10)$ & $17(14)$ & $9(9)$ & $14(13)$ \\
\hline & 2 & $90(37)$ & $86(42)$ & $50(41)$ & $40(33)$ & $46(47)$ & $40(38)$ \\
\hline & 3 & $99(41)$ & $74(36)$ & $50(41)$ & $49(41)$ & $32(32)$ & $42(40)$ \\
\hline & 4 & $20(8)$ & $15(7)$ & $11(9)$ & $9(8)$ & $9(9)$ & $6(6)$ \\
\hline & $\geq 5$ & $5(2)$ & $6(3)$ & 0 & $5(4)$ & $3(3)$ & $3(3)$ \\
\hline
\end{tabular}

Abbreviations: ECOG, Eastern Cooperative Oncology Group; $n$, number of patients per category; $N$, number of patients in population.

${ }^{\mathrm{a}} \mathrm{PBO}+\mathrm{ERL}$ arm included three Other: one Black or African American, one Missing (ex19del group), one American Indian or Alaska Native (ex21L858R group). 


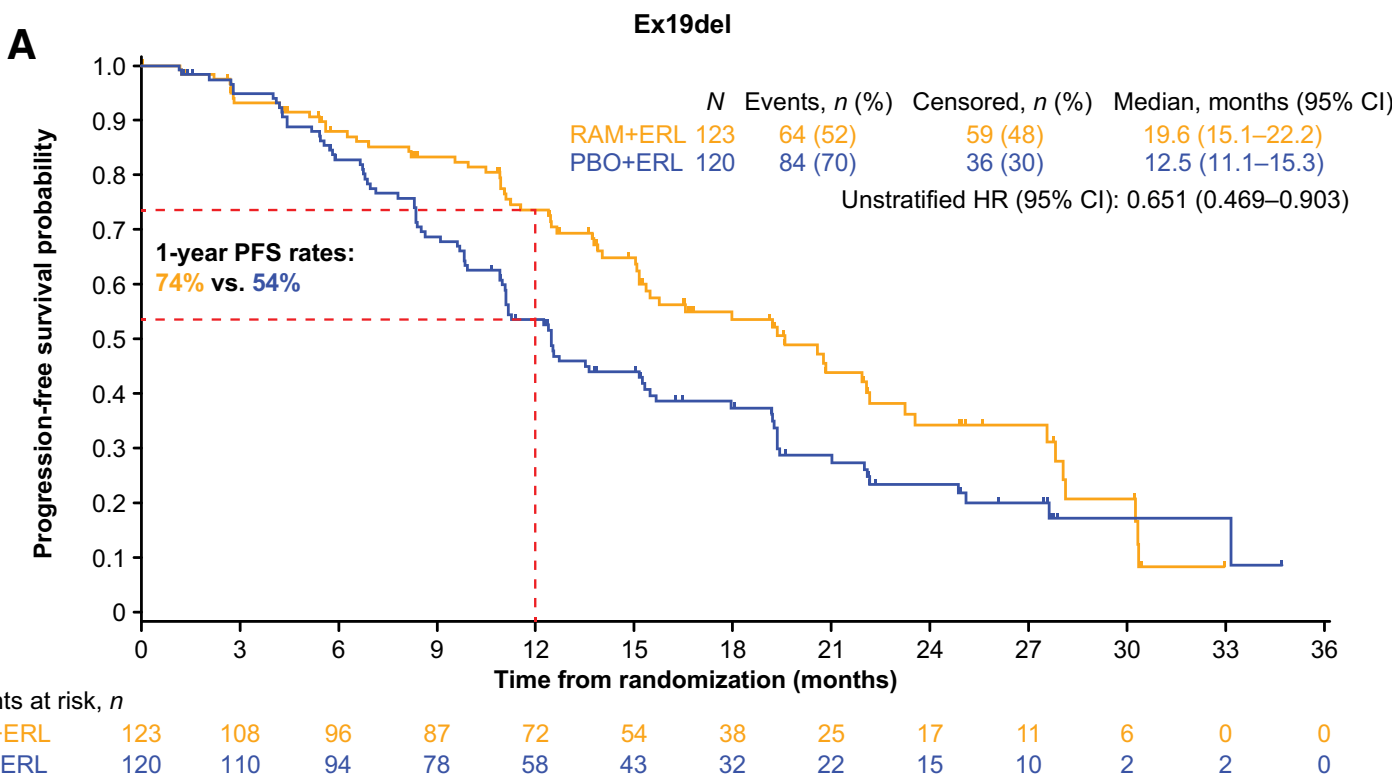

\begin{tabular}{|c|c|c|c|c|c|c|c|c|c|c|c|c|}
\hline Patient & & & & & 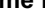 & dint & Zatu & nom & & & & \\
\hline RAM+ERL & 123 & 108 & 96 & 87 & 72 & 54 & 38 & 25 & 17 & 11 & 6 & 0 \\
\hline $\mathrm{PBO}+\mathrm{ERL}$ & 120 & 110 & 94 & 78 & 58 & 43 & 32 & 22 & 15 & 10 & 2 & 2 \\
\hline
\end{tabular}

Ex21L858R

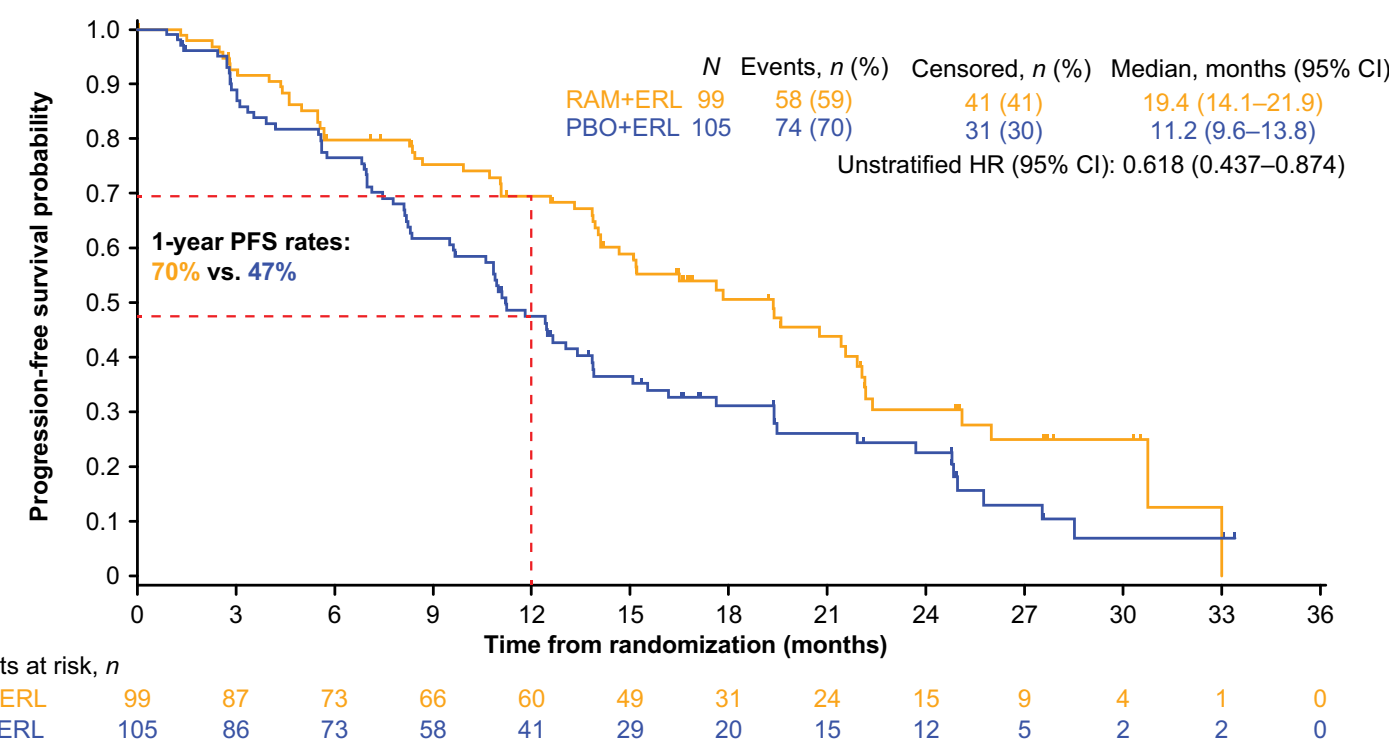

Figure 1.

Clinical endpoints by baseline activating EGFR mutation subgroup. A, Progression-free survival. Data from Nakagawa and colleagues (19). B, Duration of response. C, Progression-free survival 2. D, Time-to-chemotherapy. Abbreviations: NE, not evaluable; NR, not reached; PD, progressive disease. (Continued on the following page.)

The iOS data were immature as censoring rates were high (overall censoring rate $=82 \%$; Table 3 ). The highest rate of death was observed in the ex21L858R PBO+ERL treatment arm [ex19del: RAM+ERL 17\% (21 deaths), PBO+ERL 13\% (15); ex21L858R: RAM+ERL 16\% (16), PBO+ERL 26\% (27)]. Two-year OS rates were lowest for ex21L858R patients in the PBO+ERL treatment arm, whereas RAM+ERL-treated patients with ex21L858R reported a comparable 2-year OS rate to patients with ex19del (Table 3).
To further evaluate the impact of treatment by baseline EGFR mutation subgroups on other post-study treatment discontinuation endpoints, PFS2 and TTCT were explored. PFS2 data were immature (overall censoring rate $=69 \%$ ), with the lowest censoring rate observed for ex21L858R patients receiving PBO+ERL (58\%), indicating that most PFS2 events occurred in this subgroup. Ex21L858R patients receiving RAM+ERL continued to exhibit improved benefit through PFS2 compared with those receiving $\mathrm{PBO}+\mathrm{ERL}(\mathrm{HR}=0.600 ; 95 \% \mathrm{CI}, 0.371-0.969 ;$ Fig. 1C). The 


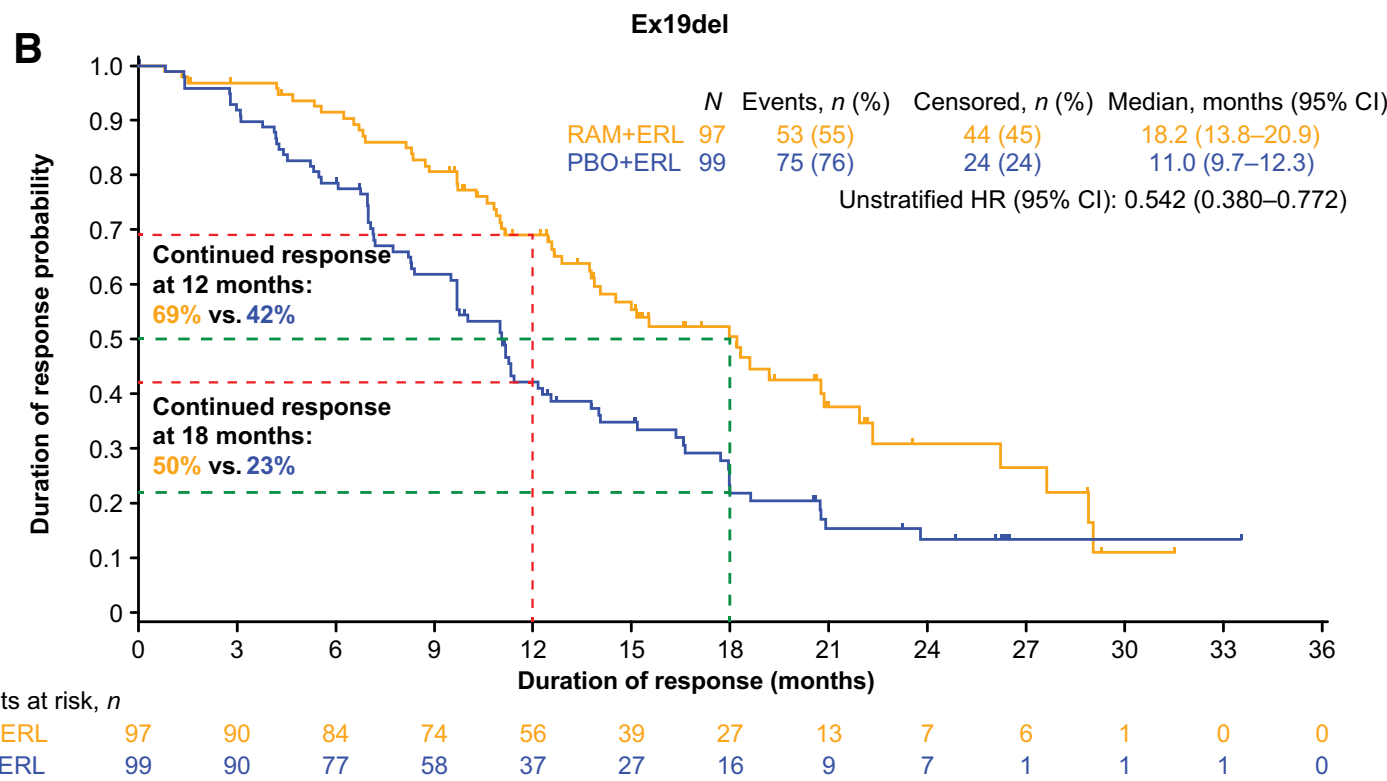

\begin{tabular}{|c|c|c|c|c|c|c|c|c|c|c|c|c|c|}
\hline \multicolumn{5}{|c|}{ Patients at risk, $n$} & \multicolumn{8}{|c|}{ (int } & \\
\hline RAM+ERL & 97 & 90 & 84 & 74 & 56 & 39 & 27 & 13 & 7 & 6 & 1 & 0 & 0 \\
\hline $\mathrm{PBO}+\mathrm{ERL}$ & 99 & 90 & 77 & 58 & 37 & 27 & 16 & 9 & 7 & 1 & 1 & 1 & 0 \\
\hline
\end{tabular}

Ex21L858R

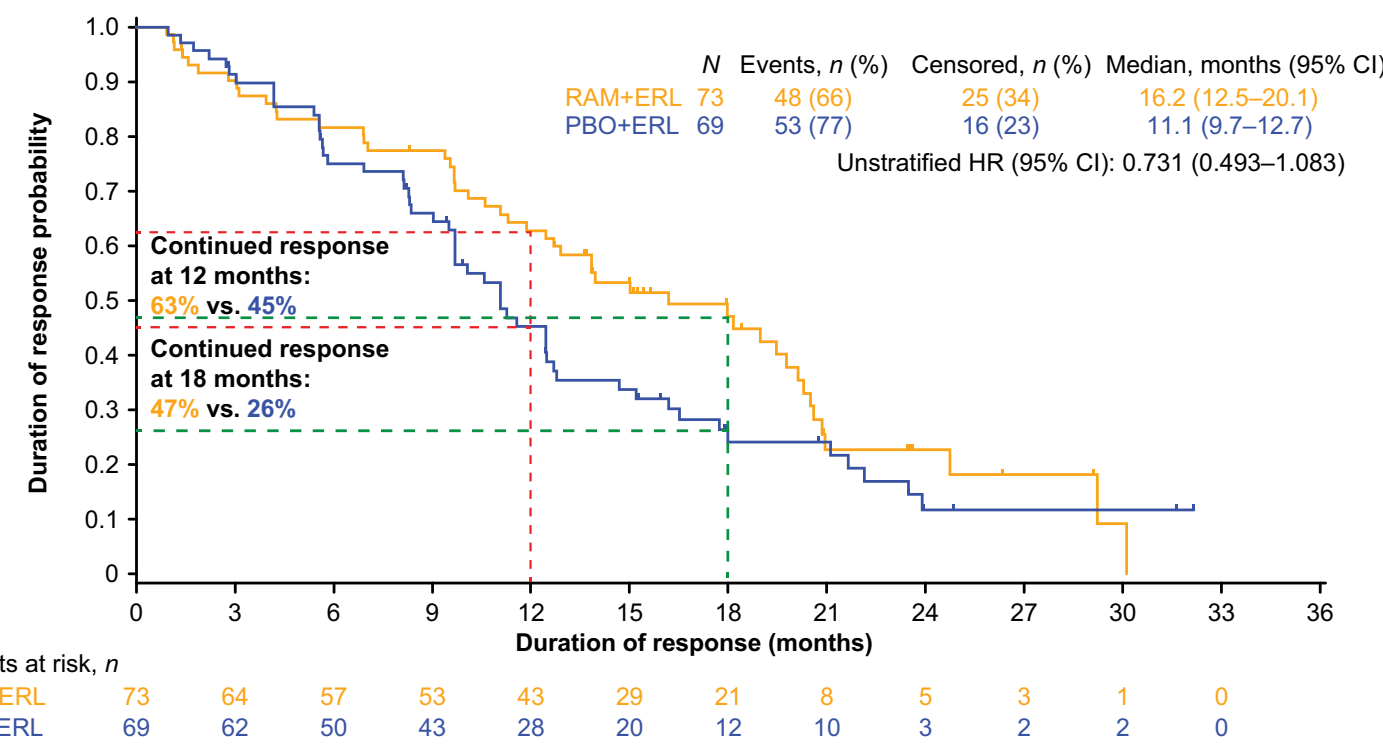

Figure 1.

(Continued.)

ex19del subgroup had similar PFS2 between treatment arms $(\mathrm{HR}=$ 0.926; 95\% CI, 0.577-1.485). For TTCT, a treatment benefit for RAM+ERL versus $\mathrm{PBO}+\mathrm{ERL}$ patients was observed for the ex21L858R subgroup ( $\mathrm{HR}=0.554 ; 95 \% \mathrm{CI}, 0.352-0.872$; censoring rate $=61 \%$; Fig. 1D). No difference between treatment arms was observed for the ex19del subgroup (HR $=1.034$; 95\% CI, 0.6821.567; censoring rate $=63 \%$ ).

Further analyses of PFS2 and TTCT both indicated a trend toward predictive effect regardless of mutation type. This trend was preserved upon adjusting for other factors via a multivariate model (interaction $P$ values: PFS2 $=0.2100$; TTCT $=0.0443$ ).

\section{Exposure}

Of those patients in the ex19del subgroup, 122 received RAM+ERL and $120 \mathrm{PBO}+\mathrm{ERL}$, and of those in the ex21L858R subgroup, 97 received $\mathrm{RAM}+\mathrm{ERL}$ and $105 \mathrm{PBO}+\mathrm{ERL}$ (Table 3). In the ex19del subgroup, among patients receiving RAM + ERL, median (minimummaximum) duration of exposure to ramucirumab (excluding 37 patients still on treatment) was $12.4(0.5-33.8)$ months and to erlotinib 15.2 (0.1-33.8) months. Among patients receiving PBO+ERL, median duration of exposure to placebo (excluding 26 patients still on treatment) was $11.0(0.5-35.4)$ months and to erlotinib 12.0 (0.4-35.5) months (Table 3 ). In the ex21L858R subgroup, among patients 


\section{C}

\section{Ex19del}

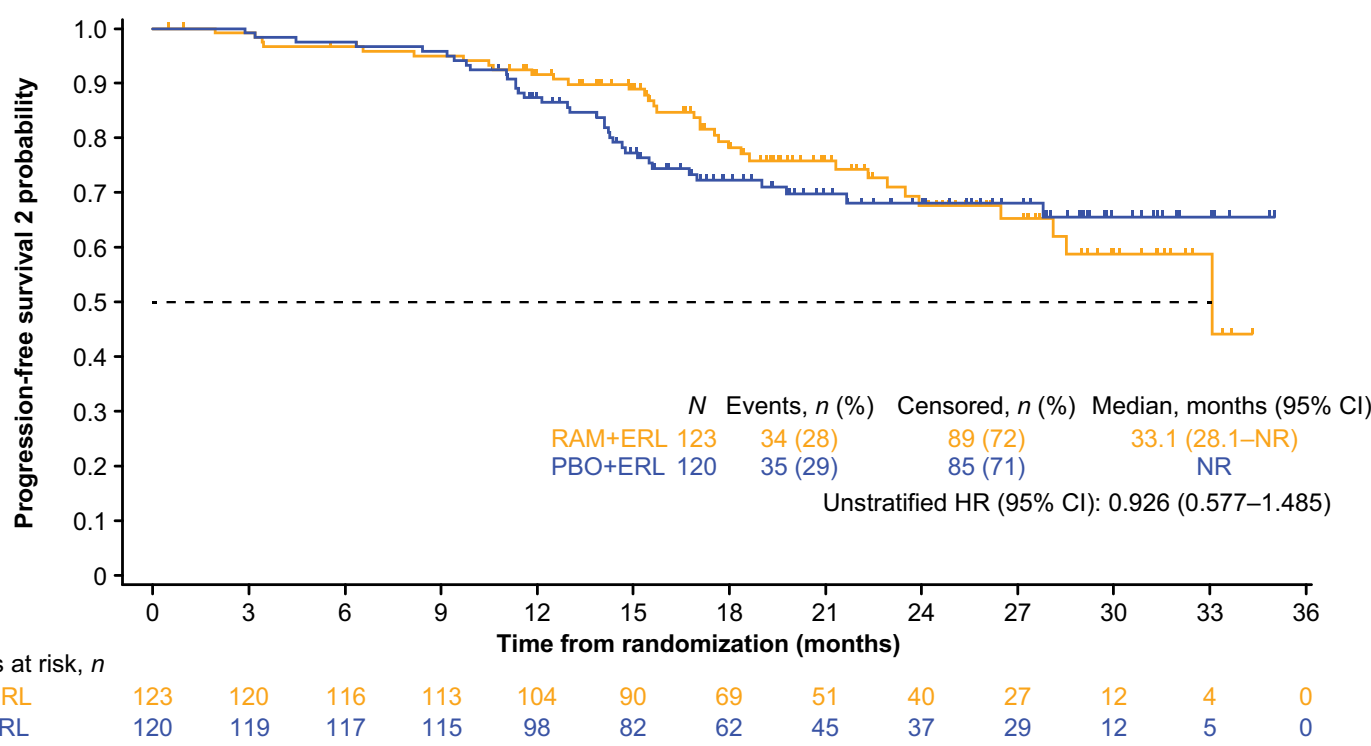

Patients at risk, $n$

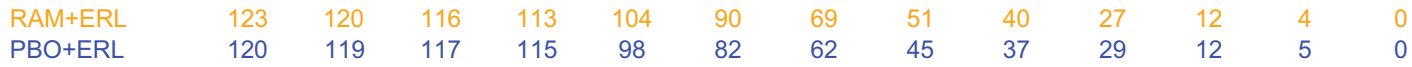

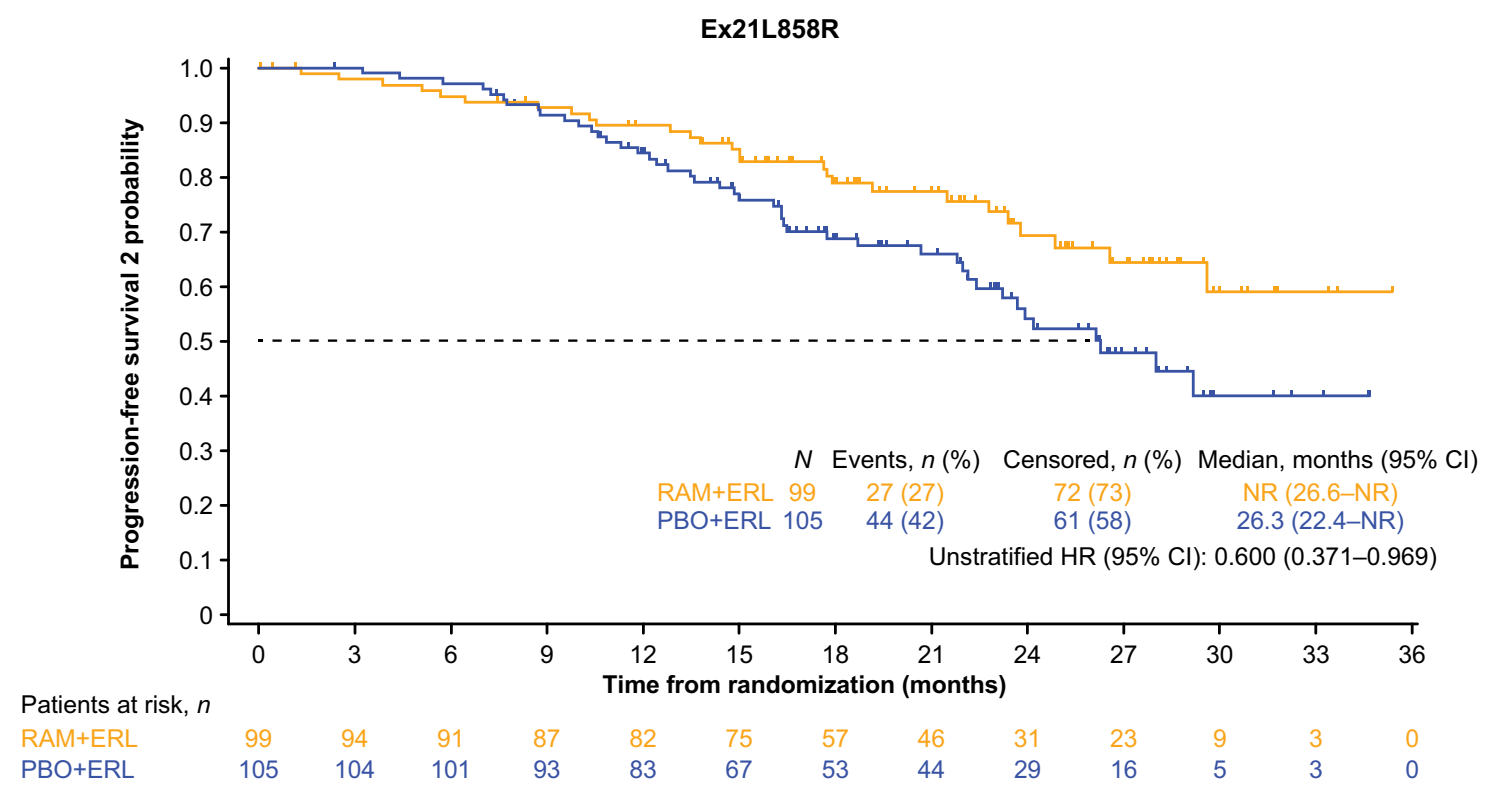

Figure 1.

(Continued.)

receiving $\mathrm{RAM}+\mathrm{ERL}$, the median duration of exposure to ramucirumab (excluding 27 patients still on treatment) was 12.7 (0.5-33.2) months and to erlotinib $15.1(0.0-33.0)$ months. Among patients receiving $\mathrm{PBO}+\mathrm{ERL}$, the median duration of exposure to placebo (excluding 27 patients still on treatment) was 8.8 (0.5-35.0) months and to erlotinib $10.4(0.8-35.1)$ months (Table 3).

\section{Safety}

All patients reported at least one treatment-emergent AE (TEAE; Supplementary Table S1). Overall, there were no clinically meaningful differences in safety findings between the mutation sub- groups, although, of note, any-grade hypertension was reported with a $\geq 10 \%$ higher incidence in the ex 21 L858R subgroup than the ex19del subgroup [50 patients (41\%) vs. 50 patients (52\%), respectively; Supplementary Table S2]. Incidence of grade 3 or higher TEAEs and serious AEs (SAE) was similar between ex19del and ex21L858R subgroups (Supplementary Table S1). Study treatment discontinuation rates due to AEs and SAEs were also similar between EGFR mutation subgroups (AEs: RAM+ERL 13\% and $13 \%$ and PBO+ERL $8 \%$ and $14 \%$, ex19del vs. ex21L858R, respectively; SAEs: RAM+ERL $3 \%$ and $6 \%$ and PBO+ERL $4 \%$ and $4 \%$, ex19del vs. ex21L858R, respectively). Death due to an AE while on 


\section{D}

Ex19del

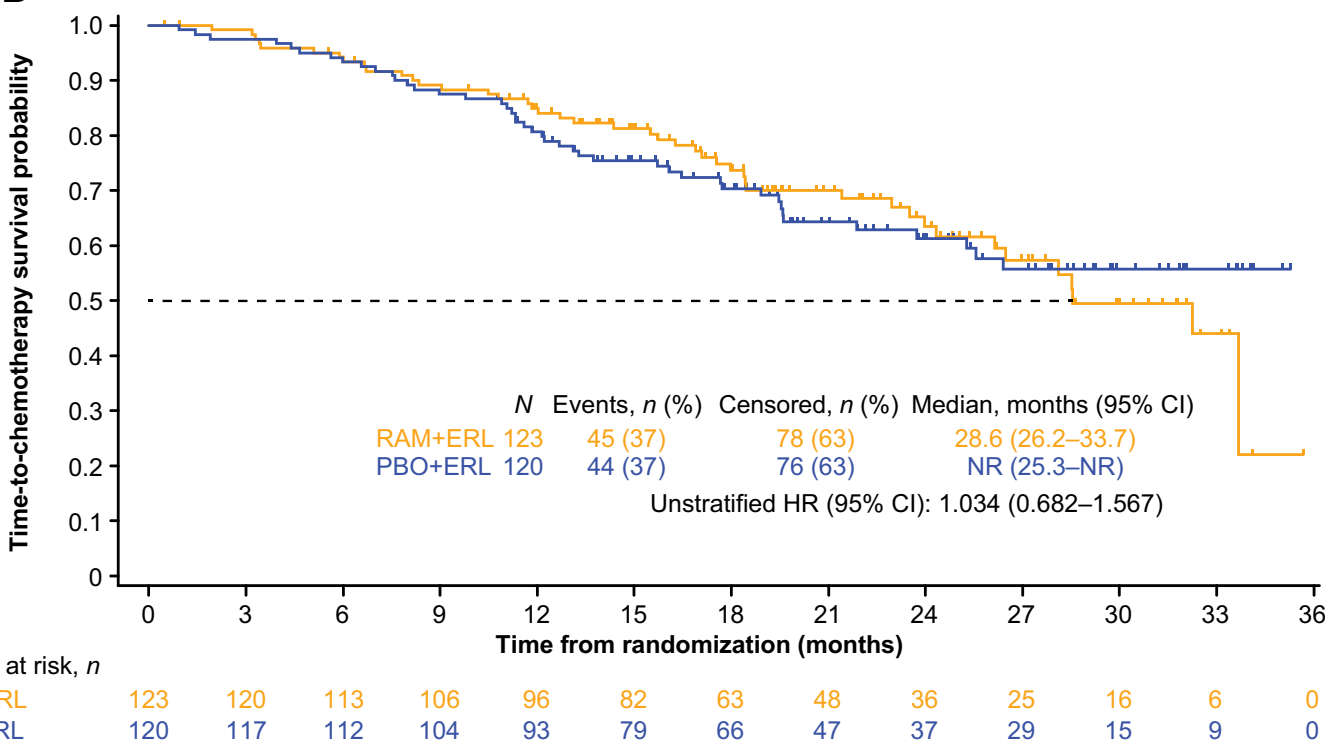

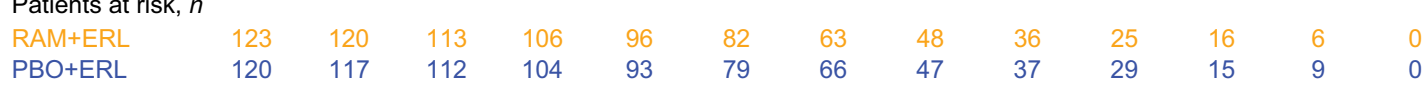

Ex21L858R

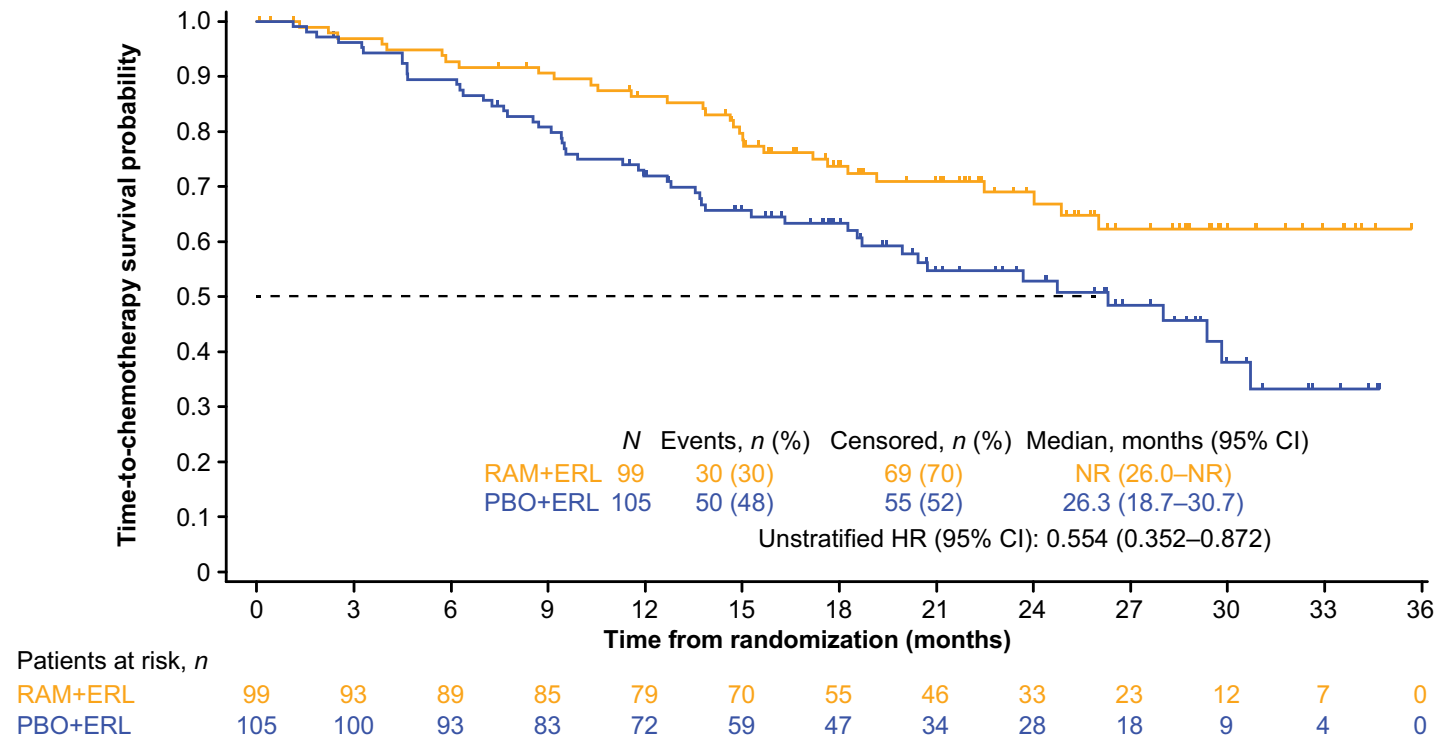

Figure 1.

(Continued.)

study treatment occurred in two RAM+ERL-treated patients with an ex21L858R mutation (one death due to hemothorax was considered related, and the other death due to encephalitis influenza was considered not related to study treatment; Supplementary Table S1).

\section{Clinical patterns of progression}

Overall, frequencies of disease progression were similar by treatment regardless of mutation type, with rates being lower for the RAM+ERL combination (ex19del: RAM+ERL 46\%, PBO+ERL 65\%; ex21L858R: RAM+ERL 49\%, PBO+ERL 64\%; Supplementary
Fig. S1). The majority of sites of progression were in the lung, followed by pleura and lymph nodes, regardless of treatment arm and EGFR mutation subgroup (Supplementary Fig. S3). CNS metastasis developed more frequently in patients with ex21L858R [6 patients in the ex21L858R subgroup (2 patients and 4 patients in the RAM+ERL and $\mathrm{PBO}+\mathrm{ERL}$ arms, respectively) and 1 patient in the ex19del subgroup $(\mathrm{PBO}+\mathrm{ERL}$ arm) $]$. The majority of patients had single-site progression; this was reported more frequently in ex21L858R patients (102 patients, 79\%) compared with ex19del patients (91 patients, $62 \%$ ). This difference was mostly driven by patients in the ex21L858R RAM+ERL treatment arm (Supplementary Fig. S4). Local progression, defined as 
Table 3. Clinical outcomes by baseline EGFR-activating mutation subgroup and by treatment arms.

\begin{tabular}{|c|c|c|c|c|}
\hline & \multicolumn{2}{|c|}{ Ex19del } & \multicolumn{2}{|c|}{ Ex21L858R } \\
\hline & RAM+ERL & PBO+ERL & $\overline{\text { RAM+ERL }}$ & PBO+ERL \\
\hline \multicolumn{5}{|l|}{ Duration of therapy ${ }^{a}$} \\
\hline$n$ & 122 & 120 & 97 & 105 \\
\hline \multicolumn{5}{|l|}{ Ramucirumab/placebo } \\
\hline Event rates, $n$ (\%) & $85(70)$ & $94(78)$ & $70(72)$ & $88(84)$ \\
\hline Censoring rates, $n$ (\%) & $37(30)$ & $26(22)$ & $27(28)$ & $17(16)$ \\
\hline Median, months (95\% Cl) & $12.4(9.4-14.8)$ & $11.0(8.5-12.4)$ & $12.7(8.0-15.1)$ & $8.8(6.8-11.1)$ \\
\hline \multicolumn{5}{|l|}{ Erlotinib } \\
\hline Event rates, $n$ (\%) & $85(70)$ & $94(78)$ & $70(72)$ & $88(84)$ \\
\hline Censoring rates, $n$ (\%) & $37(30)$ & $26(22)$ & $27(28)$ & $17(16)$ \\
\hline Median, months ( $95 \% \mathrm{Cl})$ & $15.2(12.9-19.5)$ & $12.0(10.6-13.8)$ & $15.1(12.7-19.5)$ & $10.4(7.7-12.4)$ \\
\hline \multicolumn{5}{|l|}{ Overall response rate } \\
\hline$n$ & 123 & 120 & 99 & 105 \\
\hline ORR, \% (95\% Cl) & $79(72-86)$ & $83(76-89)$ & $74(65-82)$ & $66(57-75)$ \\
\hline \multicolumn{5}{|l|}{ Disease control rate } \\
\hline$n$ & 123 & 120 & 99 & 105 \\
\hline DCR, \% (95\% Cl) & $96(92-99)$ & $96(92-99)$ & 95 (91-99) & 95 (91-99) \\
\hline \multicolumn{5}{|l|}{ Time-to-response } \\
\hline$n$ & 97 & 99 & 73 & 69 \\
\hline Median, months $(95 \% \mathrm{Cl})$ & $1.4(1.3-2.6)$ & $1.4(1.3-1.7)$ & $1.5(1.4-1.7)$ & $1.4(1.3-1.6)$ \\
\hline \multicolumn{5}{|l|}{ Duration of response } \\
\hline$n$ & 97 & 99 & 73 & 69 \\
\hline Event rates, $n(\%)$ & $53(55)$ & $75(76)$ & $48(66)$ & $53(77)$ \\
\hline Censoring rates, $n$ (\%) & $44(45)$ & $24(24)$ & $25(34)$ & $16(23)$ \\
\hline Median, months $(95 \% \mathrm{Cl})$ & $18.2(13.8-20.9)$ & $11.0(9.7-12.3)$ & $16.2(12.5-20.1)$ & $11.1(9.7-12.7)$ \\
\hline Hazard ratio $(95 \% \mathrm{Cl})$ & \multicolumn{2}{|c|}{$0.54(0.38-0.77)$} & \multicolumn{2}{|c|}{$0.73(0.49-1.08)$} \\
\hline \multicolumn{5}{|l|}{ Interim OS } \\
\hline$n$ & 123 & 120 & 99 & 105 \\
\hline Event rates, $n(\%)$ & $21(17)$ & $15(13)$ & $16(16)$ & $27(26)$ \\
\hline Censoring rates, $n$ (\%) & $102(83)$ & $105(88)$ & $83(84)$ & $78(74)$ \\
\hline Median, months & NR & NR & NR & NR \\
\hline Hazard ratio $(95 \% \mathrm{Cl})$ & $1.44(0.74-2.80)$ & & $0.61(0.33-1.14)$ & \\
\hline 1-year OS rate, \% (95\% Cl) & $94(88-97)$ & $94(88-97)$ & $92(84-96)$ & $93(86-97)$ \\
\hline 2-year OS rate, \% (95\% Cl) & $83(74-89)$ & $87(78-93)$ & $84(74-90)$ & $71(58-80)$ \\
\hline
\end{tabular}

Abbreviations: DCR, disease control rate; NR, not reached.

aDuration of therapy is censored at the last date of study treatment for patients who remain on study treatment (ex19del: $n=122$ RAM + ERL vs. 120 PBO+ERL and ex21L858R: $n=97$ RAM+ERL vs. 105 PBO+ERL patients).

increase in size of a single preexisting tumor lesion, occurred in approximately $50 \%$ of patients across treatment arms and EGFR mutation subgroups. Metastatic progression, defined as development of at least one new lesion, occurred in 52 patients (36\%) versus 53 patients $(41 \%)$ in the ex19del versus ex21L858R subgroups, respectively (Supplementary Fig. S4).

\section{Subsequent therapies}

All study treatment was to be discontinued upon RECIST v1.1defined disease progression. At time of data cutoff, 37 (30\%) and 26 (22\%) ex19del patients and $27(27 \%)$ and 17 (16\%) ex21L858R patients (RAM+ERL vs. $\mathrm{PBO}+\mathrm{ERL}$, respectively) were still on study treatment. First subsequent therapy (FST) was received by $66(54 \%)$ and 83 (69\%) ex19del patients and $54(55 \%)$ and $73(70 \%)$ ex21L858R patients (RAM+ERL vs. $\mathrm{PBO}+\mathrm{ERL}$, respectively; Supplementary Table S3). Sankey diagrams depict the flow and patterns of subsequent lines of treatment (Supplementary Fig. S5). For both EGFR mutation groups, the most commonly used FST was an EGFR-TKI, predominantly erlotinib, and to a lesser degree, osimertinib, followed by platinum-based chemotherapy. Ex19del patients treated with RAM+ERL received erlotinib as FST (52\%) more frequently compared with $\mathrm{PBO}+\mathrm{ERL}$ patients (29\%); for
ex21L858R patients, erlotinib as FST was received at similar rates in RAM+ERL (50\%) and PBO+ERL patients (42\%). Osimertinib was used more frequently as FST in ex19del patients treated with PBO+ERL (31\%) compared with RAM+ERL (14\%); for ex21L858R patients, osimertinib was received at similar rates in RAM+ERL $(17 \%)$ and $\mathrm{PBO}+\mathrm{ERL}$ patients (12\%). In ex21L858R, chemotherapy was used as FST at similar rates in patients treated with RAM+ERL and PBO+ERL ( $43 \%$ vs. $47 \%$ ), whereas more patients treated with $\mathrm{PBO}+\mathrm{ERL}$ than $\mathrm{RAM}+\mathrm{ERL}$ received chemotherapy as second subsequent therapy (SST; $66 \%$ vs. $42 \%$ ).

At time of data cutoff, approximately $35 \%$ of patients were still on FST. Nearly one third of patients who received osimertinib as FST remained on this therapy in both ex19del and ex21L858R subgroups, regardless of prior study treatment (Supplementary Table S3). As SST, osimertinib was most frequently used, regardless of prior study treatment: 29 patients (39\%) versus 16 patients $(25 \%)$ in the ex19del versus ex21L858R subgroups, respectively.

\section{Exploratory biomarker analyses}

NGS testing was performed to examine the somatic alteration landscape by ex19del or ex21L858R subgroups to gain insights on potential biological differences at baseline and mechanisms of 


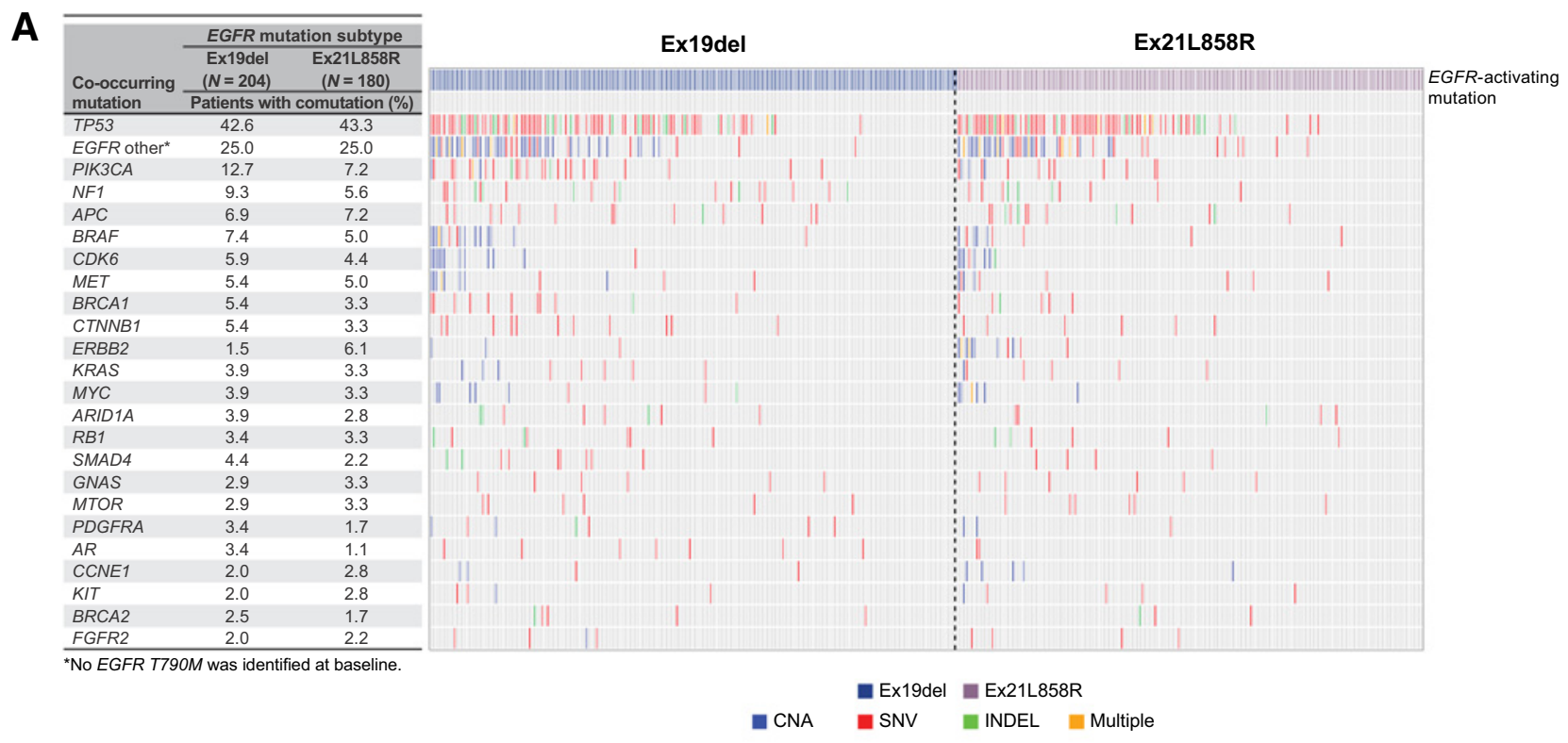

B

\begin{tabular}{|c|c|c|c|c|}
\hline \multirow{4}{*}{$\begin{array}{l}\text { Co- } \\
\text { occurring } \\
\text { mutation }\end{array}$} & \multicolumn{4}{|c|}{ EGFR mutation subtype } \\
\hline & \multicolumn{2}{|c|}{ Ex19del } & \multicolumn{2}{|c|}{ Ex21L858R } \\
\hline & $\begin{array}{l}\text { RAM+ERL } \\
(N=33)\end{array}$ & $\begin{array}{c}\text { PBO+ERL } \\
(N=53)\end{array}$ & $\begin{array}{r}\text { RAM+ER } \\
(N=34)\end{array}$ & $\begin{array}{c}\text { PBO+ERL } \\
(N=48)\end{array}$ \\
\hline & \multicolumn{4}{|c|}{ Patients with TE comutation (\%) } \\
\hline EGFR T790M & 27.3 & 34.0 & 23.5 & 27.1 \\
\hline EGFR other & 18.2 & 13.2 & 14.7 & 29.2 \\
\hline TP53 & 30.3 & 9.4 & 11.8 & 16.7 \\
\hline MET & 6.1 & 3.8 & 5.9 & 10.4 \\
\hline KRAS & 15.2 & 1.9 & 2.9 & 4.2 \\
\hline$A P C$ & 9.1 & 3.8 & 5.9 & 2.1 \\
\hline BRAF & 6.1 & 3.8 & 0 & 8.3 \\
\hline NF1 & 9.1 & 1.9 & 8.8 & 2.1 \\
\hline PIKЗСA & 3.0 & 1.9 & 0 & 12.5 \\
\hline BRCA2 & 0 & 1.9 & 5.9 & 6.3 \\
\hline ERBB2 & 3.0 & 1.9 & 2.9 & 6.3 \\
\hline FGFR2 & 6.1 & 3.8 & 2.9 & 2.1 \\
\hline ARID1A & 3.0 & 3.8 & 2.9 & 2.1 \\
\hline KIT & 6.1 & 0 & 2.9 & 2.1 \\
\hline PTEN & 3.0 & 0 & 2.9 & 4.2 \\
\hline$R B 1$ & 6.1 & 0 & 0 & 4.2 \\
\hline$A R$ & 3.0 & 0 & 0 & 4.2 \\
\hline CDK4 & 3.0 & 1.9 & 0 & 2.1 \\
\hline CDK6 & 3.0 & 0 & 0 & 4.2 \\
\hline MYC & 0 & 0 & 2.9 & 4.2 \\
\hline SMAD4 & 3.0 & 0 & 0 & 4.2 \\
\hline CCND2 & 0 & 0 & 2.9 & 2.1 \\
\hline DDR2 & 3.0 & 0 & 0 & 2.1 \\
\hline ESR1 & 3.0 & 0 & 0 & 2.1 \\
\hline FGFR1 & 3.0 & 0 & 0 & 2.1 \\
\hline GNAS & 3.0 & 0 & 2.9 & 0 \\
\hline MAP2K1 & 3.0 & 0 & 2.9 & 0 \\
\hline PDGFRA & 3.0 & 0 & 0 & 2.1 \\
\hline
\end{tabular}
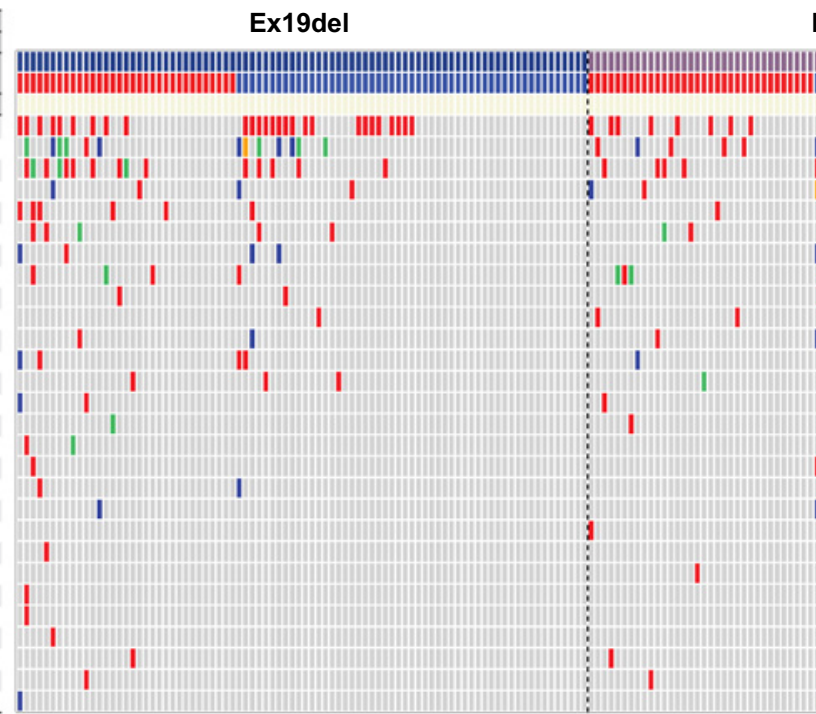

Ex21L858R

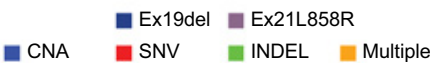

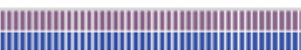
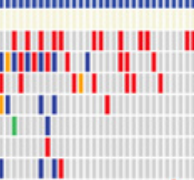

I III |

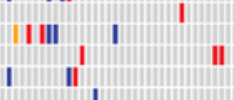

II

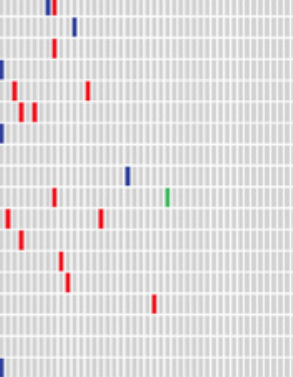

I

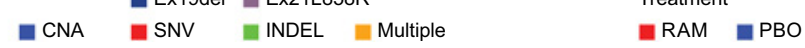

\section{Figure 2.}

Guardant360 NGS detected somatic gene alterations at baseline or treatment emergent (TE), co-occurring with ex19del or ex21L858R, and impact on outcomes. A, Baseline somatic gene alterations co-occurring with either ex19del or ex21L858R mutations as detected by Guardant360 NGS. B, Treatment-emergent somatic gene alterations co-occurring with either ex19del or ex21L858R mutations in patients with Guardant360 NGS-positive results and who had disease progression at the post-study treatment discontinuation follow-up visit. Analysis focused on those patients with at least one alteration detectable both at baseline and at disease progression; this approach, in part, controls for potential differences in tumor shedding. Abbreviations: CNA, copy-number alterations; SNV, single-nucleotide variants; wt, wild-type.

resistance. In 320 of the patients with at least one detectable somatic gene alteration, EGFR-activating mutations [ex19del: 134 (41.9\%); ex21L858R: $121(37.8 \%)]$ were detected at baseline by NGS (this was $100 \%$ concordant with local EGFR testing results); $10 \%$ of ex19del and 13\% of ex21L858R patients did not have any additional somatic gene alteration.
Concomitant gene alterations were widespread in both ex19del and ex21L858R mutated patients with NSCLC. The most frequent genes with co-occurring variants at baseline were TP53 and additional EGFR variants (Supplementary Table S4), each with similar prevalence regardless of EGFR-activating mutation subgroup (Fig. 2A). PIK3CA was higher in ex19del patients and was the only gene with co-occurring 
variants having differential prevalence $(>5 \%)$ by EGFR-activating mutation subgroup. In patients with TP53 comutation, RAM+ERL demonstrated a superior PFS treatment benefit versus PBO+ERL in both ex19del and ex21L858R subgroups (Supplementary Fig. S6). In TP53 wild-type patients, the benefit of RAM+ERL on PFS appeared more pronounced in the ex21L858R subgroup than the ex19del subgroup.

Treatment-emergent gene alterations co-occurring with either ex19del or ex21L858R mutations are presented in Fig. 2B. EGFR T790M was the most prevalent treatment-emergent gene alteration in both EGFR mutation subgroups. Other EGFR variants and TP53 were the second most prevalent comutated genes at disease progression, and prevalence varied by EGFR mutation subgroup and treatment arm. Other treatment-emergent EGFR variants were observed at highest prevalence $(29.2 \%)$ in ex21L858R PBO+ERL-treated patients, and TP53 variants were highest $(30.3 \%)$ in ex19del RAM+ERL-treated patients. The only genes with co-occurring variants having differential prevalence $(>10 \%)$ by $E G F R$-activating mutation subgroup treatment arm were KRAS (higher with RAM+ERL in ex19del patients) and PIK3CA (higher with $\mathrm{PBO}+\mathrm{ERL}$ in ex21L858R patients).

T790M rates at 30-day follow-up were similar across the two mutation subgroups and treatment arms (Supplementary Fig. S7). Among ex19del patients, emergence of T790M appeared to be delayed in those receiving RAM+ERL. T790M was only observed in ex19del patients receiving $\mathrm{RAM}+\mathrm{ERL}$ who progressed after completing at least 12 cycles of treatment compared with PBO+ERL, where emergence was seen in patients who progressed after at least four cycles of treatment. In ex21L858R patients, T790M appeared to emerge in patients after at least four cycles of treatment, independent of treatment received. Among $\mathrm{PBO}+\mathrm{ERL}-$ treated patients with postprogression TP53 detected at the 30-day follow-up, newly emergent TP53 alterations were detected as early as four cycles of treatment, independent of ex19del or ex21L858R (Supplementary Fig. S8). For RAM+ERL-treated patients, emergence of TP53 was observed in patients who completed at least four cycles for ex19del, and at least 12 cycles of treatment for ex21L858R.

\section{Discussion}

The expanding armamentarium of effective treatments for EGFR-mutated NSCLC highlights the importance of fully understanding each treatment regimen so that therapy can be tailored to individual patient and disease characteristics. There is a growing body of evidence that patients with an ex21L858R mutation have a poorer prognosis and response to treatment with EGFR-TKIs than patients with ex19del mutation.

In RELAY, RAM+ERL showed consistent treatment benefit among patients with ex19del or ex21L858R, whereas treatment outcomes were worse with ex21L858R than ex19del in those receiving PBO+ERL. Evidence suggests that different subtypes of EGFR mutation may vary in their clinical and pathologic correlations $(22,23)$. In RELAY, patient and disease characteristics at baseline, including NGS-determined comutations, were similar between EGFR mutation subgroups with the exception of ex21L858R being more prevalent among females and Asian patients. So, the subgroup of ex21L858R patients did not appear to be enriched with more poor prognostic features than the subgroup of ex19del patients.

Patterns of progression were reported at similar rates between the two mutation subgroups, with the exception of CNS metastases being more frequently reported as site of first progression in patients with
ex21L858R. This could indicate a more aggressive phenotype of ex21L858R tumors, although the small number of events limits the strength of this observation.

The magnitude of PFS benefit with RAM+ERL observed for patients with ex21L858R was similar to that of RAM+ERL-treated patients with ex19del (19). RAM+ERL-treated patients with ex21L858R also had treatment benefits in ORR, DOR, PFS2, TTCT, and iOS; the worst outcomes were observed in PBO+ERL-treated patients with ex21L858R. Analyses evaluating TTCT showed that, despite high censoring, use of chemotherapy was significantly delayed with RAM+ERL versus $\mathrm{PBO}+\mathrm{ERL}$ in ex21L858R patients but not ex19del patients. Subsequent therapies received and presence of treatment-emergent T790M and/or TP53 mutations may have had a role in the TTCT results observed. Although a predictive effect of mutation type on PFS was not apparent, further analyses of PFS2 and TTCT both indicated a trend toward predictive effect regardless of mutation type on benefit of RAM+ERL. Given limited event counts and data immaturity for post-progression outcomes and ex19del being more common in Western patients, who were underrepresented in RELAY (28\% were Caucasian), the results are to be interpreted with caution. In general, there were no meaningful differences in the safety profiles of RAM+ERL by activating mutation subgroups.

The underlying reasons for the differential efficacy between ex19del and ex21L858R mutation subtypes being treated with EGFR-TKI monotherapy are not fully understood (12). One suggestion is that ex21L858R is less efficiently inhibited by EGFR-TKIs than ex19del.

In vitro studies have shown that gefitinib inhibited EGFR phosphorylation, a measure of kinase inhibitor potency, to a lesser degree in cancer cells with ex21L858R than with ex19del mutation (24), whereas kinetic analysis has demonstrated ex21L858R to be less sensitive than ex19del to erlotinib inhibition (25). These findings offer some rationale for the difference in outcomes between the EGFR mutation subtypes when treated with single-agent EGFR-TKI.

Interestingly, a consistent PFS benefit between ex19del and ex21L858R groups has also been reported in patients receiving erlotinib and bevacizumab (Table 1; refs. 16-18), and meta-analyses of EGFR-TKI and antiangiogenic agent combinations $(26,27)$, suggesting that addition of an antiangiogenic agent appears to abrogate the differential efficacy observed in ex21L858R compared with ex19del patients when treated with EGFR-TKI monotherapy. Unfortunately, a comprehensive understanding of the underlying biological mechanisms that could support the clinical observations with ex19del and ex21L858R mutations is currently lacking, and so it is unknown if this is related to dual EGFR/VEGF pathway inhibition. On the basis of the observation that ex21L858R patients receiving high-dose EGFRTKI treatment have improved outcomes compared with those receiving standard-dose EGFR-TKI (28), it could be hypothesized that addition of an antiangiogenic agent might lead to increased and sustained high concentrations and uptake of EGFR-TKIs by normalizing blood flow through tumor blood vessels $(25,29,30)$. Further preclinical studies are needed to elucidate biological differences between ex19del and ex21L858R mutations.

The wide range of preexisting co-occurring and treatmentemergent genetic alterations underscore the fact that EGFR-mutant lung cancer is a heterogeneous disease, which might contribute to the differential sensitivity and varied responses achieved upon EGFR-TKI treatment. Concurrent TP53 mutations have been shown to be a negative prognostic factor and associated with poorer outcomes in patients treated with EGFR-TKIs (31). A role for TP53 in angiogenesis has also been established, and multiple studies have suggested that presence of a TP53 mutation is associated with VEGF pathway 
upregulation and may be predictive of clinical sensitivity to antiangiogenic therapies in several tumor types (32-35). In RELAY, the prevalence of TP53 mutations at baseline ( $43 \%$ vs. $43 \%$ ) and at time of disease progression (17\% vs. $15 \%)$ was similar in ex19del and ex21L858R subgroups. Baseline TP53 mutation was associated with superior outcomes for RAM+ERL in both ex19del and ex21L858R subgroups, whereas in TP53 wild-type patients, the benefit of RAM+ERL appeared more pronounced in the ex21L858R than the ex19del subgroup. At disease progression, treatment-emergent TP53 variants were most prevalent in ex19del RAM+ERL-treated patients, and additional experimentation is needed to determine the relevance and if it is related to mechanisms of acquired resistance.

Further, treatment-emergent $T 790 M$ as a mechanism of resistance is reported to occur more frequently in ex19del than ex21L858R patients $(36,37)$. In RELAY, no T790M was identified in circulating tumor DNA plasma samples at baseline; however, T790M was, as expected, the most prevalent genetic alteration poststudy treatment discontinuation. EGFR T790M mutation rate at progression was similar between treatment arms and by mutation type (Supplementary Fig. S1), suggesting that addition of ramucirumab to erlotinib does not alter the T790M resistance mechanism pathway. In ex19del patients, the emergence of T790M may be delayed in those receiving RAM+ERL, whereas this was not observed in ex21L858R patients. However, as median PFS times were similar for RAM+ERL-treated ex19del and ex21L858R patients, the difference in the timing of T790M emergence does not appear to contribute to the enhanced benefit observed in ex21L858R patients upon addition of ramucirumab to erlotinib treatment. Subsequent treatment with an agent that targets the EGFR T790M mutation, such as osimertinib, could further delay disease progression and TTCT for the considerable proportion of patients who acquire the EGFR T790M mutation. Indeed, in this study, osimertinib was used as post-discontinuation therapy across subsequent lines of therapy (Supplementary Table S3).

We acknowledge a number of limitations in our study. RELAY was not sufficiently powered for subgroup analyses of ex19del and ex21L858R, although these were prespecified, and a randomization factor. In addition, results of the exploratory biomarker analyses should be viewed with caution because of low patient numbers at varying time points. All NGS analyses were performed on plasma samples only. As a consequence, some important mechanisms of resistance, such as histological transformation and copy-number alterations (which are more difficult to capture in plasma) have not been explored optimally. Differences in prevalence and timing of treatment-emergent mutations between treatment arms (e.g., delay in emergence of T790M or TP53) are possibly biased toward early progressors, as patients for whom no post-discontinuation plasma is available yet are those still benefiting from treatment. The final NGS analysis at time of OS maturity should provide a clearer understanding of the impact of treatmentemergent alterations.

Analyses of the EGFR mutation subgroups in RELAY is for the purposes of hypothesis generation and reflects an attempt to better understand the heterogeneity of the disease. We believe that understanding the potential impact of differences between ex19del and ex21L858R patients is clinically relevant and could have several potentially significant clinical and research implications. Findings will be useful for counseling patients and could help inform treatment decisions. Our data demonstrate that ex19del and ex21L8585R mutations have different prognostic and predictive roles dependent on the therapy used and are therefore an important stratification factor in clinical trials. Further drug development, particularly for tumors with ex21L858R that have a poorer response to EGFR-TKI monotherapy, remains important. Further prospective studies are warranted to confirm the role of combined EGFR-TKI and antiangiogenic treatment in patients with ex21L858R mutations. The recently started WJOG phase III study of erlotinib plus ramucirumab versus osimertinib in advanced NSCLC with EGFR ex21L858R mutation (REVOL858R; jRCTs051200142) will help address this question.

In summary, RELAY demonstrates that RAM+ERL has significant clinical benefit for both EGFR ex19del and ex21L858R NSCLC, supporting this regimen as suitable for patients with either of these EGFR mutation types. However, as ex21L858R-positive patients historically have poorer outcomes relative to ex19del in response to EGFR-TKI monotherapies, this subgroup of EGFRmutant NSCLC may be a higher priority for addition of an antiangiogenic to an EGFR-TKI. Our findings provide evidence that patients with EGFR ex19del and ex21L8585R mutations do not have a heterogeneous response to EGFR-TKI targeted therapy when combined with ramucirumab. These findings may help clinicians provide a personalized treatment strategy and allow treatment decisions to be made on a more rational basis.

\section{Authors' Disclosure}

K. Nakagawa reports grants and personal fees from AstraZeneca K.K., MSD K.K., Nippon Boehringer Ingelheim Co., Ltd., Novartis Pharma K.K., Bristol Myers Squibb Company, Chugai Pharmaceutical Co., Ltd., Daiichi Sankyo Co., Ltd., and Merck Serono Co., Ltd./Merck Biopharma Co., Ltd., as well as grants, personal fees, and other support from Astellas Pharma Inc., Ono Pharmaceutical Co., Ltd.; Pfizer Japan Inc., and Eli Lilly Japan K.K. during the conduct of the study. K. Nakagawa also reports personal fees from MEDICUS SHUPPAN, Publishers Co., Ltd., Care Net, Inc., Medical Review Co., Ltd., Roche Diagnostics K.K., Medical Mobile Communications Co., Ltd., 3H Clinical Trial Inc., Nichi-Iko Pharmaceutical Co., Ltd., Yodosha Co., Ltd., Nikkei Business Publications, Inc., Thermo Fisher Scientific K.K., Yomiuri Telecasting Corporation, Nippon Kayaku Co., Ltd., Hisamitsu Pharmaceutical Co., Inc., NANZANDO Co., Ltd., and Amgen Inc.; personal fees and other support from KYORIN Pharmaceutical Co., Ltd.; grants, personal fees, and other support from Takeda Pharmaceutical Co., Ltd.; grants and personal fees from Taiho Pharmaceutical Co., Ltd. and AbbVie Inc., Kyowa Kirin Co., Ltd., and Bayer Yakuhin, Ltd.; and grants from SymBio Pharmaceuticals Limited, ICON Japan K.K., PAREXEL International Corp., Kissei Pharmaceutical Co., Ltd., EPS Corporation, Syneos Health, Pfizer R\&D Japan G.K., A2 Healthcare Corp., IQVIA Services JAPAN K.K., Eisai Co., Ltd., CMIC Shift Zero K.K., EPS International Co., Ltd., Otsuka Pharmaceutical Co., Ltd., PRA HEALTHSCIENCES, Covance Japan Inc., Medical Research Support, Sanofi K.K., PPD-SNBL K.K., Japan Clinical Research Operations, Sysmex Corporation, AbbVie Inc., Mochida Pharmaceutical Co., Ltd., and GlaxoSmithKline K.K. outside the submitted work. E. Nadal reports personal fees and non-financial support from Lilly during the conduct of the study. E. Nadal also reports grants, personal fees, and non-financial support from Bristol Myers Squibb, Roche, and Pfizer; personal fees and nonfinancial support from Merck Sharp \& Dohme and Amgen, AstraZeneca, and Bayer; and grants and personal fees from Merck Serono and Takeda outside the submitted work. E.B. Garon reports grants from Eli Lilly during the conduct of the study. E.B. Garon also reports grants from AstraZeneca, Eli Lilly, Genentech, Iovance Biotherapeutics, Mirati Therapeutics, Neon, and Dynavax; grants and personal fees from EMD Serono, ABL-Bio, Bristol Myers Squibb, Boehringer Ingelheim, and Merck; and personal fees from Dracen Pharmaceuticals, Eisai, GlaxoSmithKline, Natera, Novartis, Regeneron, Sanofi, Shionogi, and Xilio outside the submitted work. T. Seto reports grants from Eli Lilly Japan during the conduct of the study. T. Seto also reports grants and personal fees from Chugai Pharmaceutical, Daiichi Sankyo, Eli Lilly Japan, MSD, Novartis Pharma, Pfizer Japan, Takeda Pharmaceutical, and AbbVie; grants from Kissei Pharmaceutical, LOXO Oncology, and Merck Biopharma; and personal fees from AstraZeneca, Bristol Myers Squibb, Covidien Japan, Kyowa Hakko Kirin, Mochida Pharmaceutical, Nippon Boehringer Ingelheim, Ono Pharmaceutical, Taiho Pharmaceutical, Thermo Fisher Scientific, and Precision Medicine Asia outside the submitted work. N. Yamamoto reports personal fees from 
AstraZeneca, MSD K.K., Ono Pharmaceutical Co., Ltd., Thermo Fisher Scientific, and Daiichi Sankyo Co., Ltd.; grants and personal fees from Taiho Pharmaceutical Co., Ltd., Chugai Pharmaceutical Co., Ltd., and Boehringer Ingelheim; and personal fees from Takeda Pharmaceutical Co., Ltd., Eli Lilly Japan K.K., Novartis, Pfizer Inc., Bristol Myers Squibb, NIPPON KAYAKU, GlaxoSmithKline K.K., Sanofi K.K., Hisamitsu Pharmaceutical Co. Inc., and Merck Biopharma outside the submitted work. K. Park reports other support from Eli Lilly outside the submitted work. J.-Y. Shih reports personal fees from AstraZeneca, Boehringer Ingelheim, Eli Lilly, Pfizer, Novartis, MSD, Chugai Pharma, Ono Pharmaceutical, BMS, and AbbVie, as well as grants and personal fees from Roche outside the submitted work. L. Paz-Ares reports personal fees from Amgen, AstraZeneca, Bayer, BMS, GSK, Ipsen, Janssen, Lilly, Merck Serono, Mirati, MSD, Novartis, Pfizer, Pharmamar, Roche, Sanofi, Takeda, and Tesaro outside the submitted work; L. Paz-Ares also has a patent for Altum Sequency issued. B. Frimodt-Moller reports employment with Eli Lilly. A.H. Zimmermann reports employment with Eli Lilly and Company and ownership of Eli Lilly stock. S. Wijayawardana reports personal fees from Eli Lilly and Company outside the submitted work. C. Visseren-Grul reports other support from Eli Lilly during the conduct of the study. M. Reck reports personal fees from Amgen, AstraZeneca, BMS, Boehringer Ingelheim, Lilly, Merck, Mirati, MSD, Novartis, Pfizer, Roche, and Sanofi outside the submitted work.

\section{Data Sharing Statement}

Lilly provides access to all individual participant data collected during the trial, after anonymization, with the exception of pharmacokinetic or genetic data. Data are available to request 6 months after the indication studied has been approved in the US and $\mathrm{EU}$ and after primary publication acceptance, whichever is later. No expiration date of data requests is currently set once data are made available. Access is provided after a proposal has been approved by an independent review committee identified for this purpose and after receipt of a signed data sharing agreement. Data and documents, including the study protocol, statistical analysis plan, clinical study report, and blank or annotated case report forms, will be provided in a secure data sharing environment. For details on submitting a request, see the instructions provided at www.vivli.org.

\section{References}

1. Hsu WH, Yang JC, Mok TS, Loong HH. Overview of current systemic management of EGFR-mutant NSCLC. Ann Oncol 2018;29:i3-i9.

2. Midha A, Dearden S, McCormack R. EGFR mutation incidence in nonsmall-cell lung cancer of adenocarcinoma histology: a systematic review and global map by ethnicity (mutMapII). Am J Cancer Res 2015;5:2892911.

3. Passaro A, Prelaj A, Bonanno L, Tiseo M, Tuzi A, Proto C, et al. Activity of EGFR TKIs in Caucasian patients with NSCLC harboring potentially sensitive uncommon EGFR mutations. Clin Lung Cancer 2019;20:e186-e94.

4. Murray S, Dahabreh IJ, Linardou H, Manoloukos M, Bafaloukos D, Kosmidis P. Somatic mutations of the tyrosine kinase domain of epidermal growth factor receptor and tyrosine kinase inhibitor response to TKIs in non-small cell lung cancer: an analytical database. J Thorac Oncol 2008;3: $832-9$.

5. Zhang YL, Yuan JQ, Wang KF, Fu XH, Han XR, Threapleton D, et al. The prevalence of EGFR mutation in patients with non-small cell lung cancer: a systematic review and meta-analysis. Oncotarget 2016;7:78985-93.

6. Graham RP, Treece AL, Lindeman NI, Vasalos P, Shan M, Jennings LJ, et al. Worldwide frequency of commonly detected EGFR mutations. Arch Pathol Lab Med 2018;142:163-7.

7. Hong W, Wu Q, Zhang J, Zhou Y. Prognostic value of EGFR 19-del and 21L858R mutations in patients with non-small cell lung cancer. Oncol Lett 2019;18: 3887-95.

8. Jiang H, Zhu M, Li Y, Li Q. Association between EGFR exon 19 or exon 21 mutations and survival rates after first-line EGFR-TKI treatment in patients with non-small cell lung cancer. Mol Clin Oncol 2019;11:301-8.

9. Saad ED, Zalcberg JR, Peron J, Coart E, Burzykowski T, Buyse M. Understanding and communicating measures of treatment effect on survival: can we do better? J Natl Cancer Inst 2018;110:232-40.

10. Sashegyi A, Ferry D. On the interpretation of the hazard ratio and communication of survival benefit. Oncologist 2017;22:484-6.

\section{Authors' Contributions}

K. Nakagawa: Conceptualization, writing-review and editing. E. Nadal: Resources, data curation, writing-review and editing. E.B. Garon: Conceptualization, resources, data curation, writing-review and editing. M. Nishio: Resources, data curation, writing-review and editing. T. Seto: Resources, data curation, writing-review and editing. N. Yamamoto: Resources, data curation, writing-review and editing. K. Park: Resources, data curation, writing-review and editing. J.-Y. Shih: Resources, data curation, writing-review and editing. L. Paz-Ares: Writing-review and editing. B. Frimodt-Moller: Writing-review and editing. A.H. Zimmermann: Conceptualization, validation, writing-review and editing. S. Wijayawardana: Validation, writing-review and editing. C. Visseren-Grul: Project administration, writingreview and editing. M. Reck: Conceptualization, resources, data curation, writingreview and editing.

\section{Acknowledgments}

The RELAY clinical trial was sponsored and funded by Eli Lilly and Company. The authors are grateful to the physicians, clinical staff, caregivers, and especially patients for their invaluable contributions to this work. Medical writing assistance was provided by Susan P. Whitman, a full-time employee of Eli Lilly and Company, and John Bilbruck of ProScribe - Envision Pharma Group, and was funded by Eli Lilly Shawn Estrem, a full-time employee of Eli Lilly and Company, provided biomarker analyses and interpretations. Malika Mahoui, Hilary Graham, Olga N. Lipkovich, and Donghui Li, full-time employees of Eli Lilly and Company, provided statistical analyses and Sankey diagrams. The contributing RELAY investigators are listed in the Supplementary Materials. Eli Lilly and Company sponsored this clinical trial, provided study drugs, and participated in regulatory and ethics approval, safety monitoring, data collection, and statistical analyses.

The costs of publication of this article were defrayed in part by the payment of page charges. This article must therefore be hereby marked advertisement in accordance with 18 U.S.C. Section 1734 solely to indicate this fact.

Received February 3, 2021; revised April 8, 2021; accepted July 19, 2021; published first July $21,2021$.

11. Schnipper LE, Davidson NE, Wollins DS, Blayney DW, Dicker AP, Ganz PA et al. Updating the American Society of Clinical Oncology Value Framework: revisions and reflections in response to comments received. J Clin Oncol 2016;34: 2925-34

12. Sheng M, Wang F, Zhao Y, Li S, Wang X, Shou T, et al. Comparison of clinical outcomes of patients with non-small-cell lung cancer harbouring epiderma growth factor receptor exon 19 or exon 21 mutations after tyrosine kinase inhibitors treatment: a meta-analysis. Eur J Clin Pharmacol 2016;72:1-11.

13. Zhang Y, Sheng J, Kang S, Fang W, Yan Y, Hu Z, et al. Patients with exon 19 deletion were associated with longer progression-free survival compared to those with L858R mutation after first-line EGFR-TKIs for advanced non-small cell lung cancer: a meta-analysis. PLoS One 2014;9:e107161.

14. Arriola E, Garcia Gómez R, Diz P, Majem M, Martinez Aguillo M, Valdivia J, et al. Clinical management and outcome of patients with advanced NSCLC carrying EGFR mutations in Spain. BMC Cancer 2018;18:106.

15. Byers LA, Heymach JV. Dual targeting of the vascular endothelial growth factor and epidermal growth factor receptor pathways: rationale and clinical applications for non-small-cell lung cancer. Clin Lung Cancer 2007;8:S79-85.

16. Seto T, Kato T, Nishio M, Goto K, Atagi S, Hosomi Y, et al. Erlotinib alone or with bevacizumab as first-line therapy in patients with advanced nonsquamous non-small-cell lung cancer harbouring EGFR mutations (JO25567) an open-label, randomised, multicentre, phase 2 study. Lancet Oncol 2014;15 1236-44.

17. Saito H, Fukuhara T, Furuya N, Watanabe K, Sugawara S, Iwasawa S, et al. Erlotinib plus bevacizumab versus erlotinib alone in patients with EGFR-positive advanced non-squamous non-small-cell lung cancer (NEJ026): interim analysis of an open-label, randomised, multicentre, phase 3 trial. Lancet Oncol 2019;20: 625-35.

18. Zhou Q, Wu Y-L, Cheng Y, Liu Y, Chen G, Cui J, et al. CTONG 1509: phase II study of bevacizumab with or without erlotinib in untreated Chinese patients with advanced EGFR-mutated NSCLC. Ann Oncol 2019;30:v603. 
19. Nakagawa K, Garon EB, Seto T, Nishio M, Ponce Aix S, Paz-Ares L, et al. Ramucirumab plus erlotinib in patients with untreated, EGFR-mutated, advanced non-small-cell lung cancer (RELAY): a randomised, double-blind, placebo-controlled, phase 3 trial. Lancet Oncol 2019;20:1655-69.

20. Le X, Nilsson M, Goldman J, Reck M, Nakagawa K, Kato T, et al. Dual EGFRVEGF pathway inhibition: a promising strategy for patients with EGFR-mutant NSCLC. J Thorac Oncol 2021;16:205-15.

21. Thompson JC, Yee SS, Troxel AB, Savitch SL, Fan R, Balli D, et al. Detection of therapeutically targetable driver and resistance mutations in lung cancer patients by next-generation sequencing of cell-free circulating tumor DNA. Clin Cancer Res 2016;22:5772-82.

22. Isaka T, Yokose T, Ito H, Nagata M, Furumoto H, Nishii T, et al. Correlations between the EGFR mutation status and clinicopathological features of clinical stage I lung adenocarcinoma. Medicine (Baltimore) 2015;94:e1784.

23. Zhou X, Cai L, Liu J, Hua X, Zhang Y, Zhao H, et al. Analyzing EGFR mutations and their association with clinicopathological characteristics and prognosis of patients with lung adenocarcinoma. Oncol Lett 2018; $16: 362-70$.

24. Zhu JQ, Zhong WZ, Zhang GC, Li R, Zhang XC, Guo AL, et al. Better survival with EGFR exon 19 than exon 21 mutations in gefitinib-treated non-small cell lung cancer patients is due to differential inhibition of downstream signals. Cancer Lett 2008;265:307-17.

25. Carey KD, Garton AJ, Romero MS, Kahler J, Thomson S, Ross S, et al. Kinetic analysis of epidermal growth factor receptor somatic mutant proteins shows increased sensitivity to the epidermal growth factor receptor tyrosine kinase inhibitor, erlotinib. Cancer Res 2006;66:8163-71.

26. Deng Z, Qin Y, Liu Y, Zhang Y, Lu Y. Role of antiangiogenic agents combined with EGFR tyrosine kinase inhibitors in treatment-naive lung cancer: a metaanalysis. Clin Lung Cancer 2021;22:e70-e83.

27. Chen F, Chen N, Yu Y, Cui J. Efficacy and safety of epidermal growth factor receptor (EGFR) inhibitors plus antiangiogenic agents as first-line treatments for patients with advanced EGFR-mutated non-small cell lung cancer: a metaanalysis. Front Oncol 2020;10:904

28. Li X, Zhang L, Jiang D, Wang Y, Zang A, Ding C, et al. Routine-dose and highdose icotinib in patients with advanced non-small cell lung cancer harboring EGFR exon 21-L858R mutation: the randomized, phase II, INCREASE trial. Clin Cancer Res 2020;26:3162-71.

29. Chauhan VP, Stylianopoulos T, Martin JD, Popovic Z, Chen O, Kamoun WS, et al. Normalization of tumour blood vessels improves the delivery of nanomedicines in a size-dependent manner. Nat Nanotechnol 2012;7: 383-8.

30. Chatterjee S, Wieczorek C, Schottle J, Siobal M, Hinze Y, Franz T, et al. Transient antiangiogenic treatment improves delivery of cytotoxic compounds and therapeutic outcome in lung cancer. Cancer Res 2014;74:2816-24.

31. Qin K, Hou H, Liang Y, Zhang X. Prognostic value of TP53 concurrent mutations for EGFR- TKIs and ALK-TKIs based targeted therapy in advanced non-small cell lung cancer: a meta-analysis. BMC Cancer 2020;20:328.
32. Li AM, Boichard A, Kurzrock R. Mutated TP53 is a marker of increased VEGF expression: analysis of 7,525 pan-cancer tissues. Cancer Biol Ther 2020;21:95-100.

33. Schwaederlé M, Lazar V, Validire P, Hansson J, Lacroix L, Soria JC, et al. VEGF-A expression correlates with TP53 mutations in non-small cell lung cancer: implications for antiangiogenesis therapy. Cancer Res 2015;75:1187-90.

34. Narendran A, Ganjavi H, Morson N, Connor A, Barlow JW, Keystone E, et al Mutant p53 in bone marrow stromal cells increases VEGF expression and supports leukemia cell growth. Exp Hematol 2003;31:693-701.

35. Wheler JJ, Janku F, Naing A, Li Y, Stephen B, Zinner R, et al. TP53 alterations correlate with response to VEGF/VEGFR inhibitors: implications for targeted therapeutics. Mol Cancer Ther 2016;15:2475-85.

36. Pao W, Miller VA, Politi KA, Riely GJ, Somwar R, Zakowski MF, et al. Acquired resistance of lung adenocarcinomas to gefitinib or erlotinib is associated with a second mutation in the EGFR kinase domain. PLoS Med 2005;2:e73.

37. Kobayashi S, Boggon TJ, Dayaram T, Janne PA, Kocher O, Meyerson M, et al. EGFR mutation and resistance of non-small-cell lung cancer to gefitinib. N Engl J Med 2005;352:786-92.

38. Soria JC, Ohe Y, Vansteenkiste J, Reungwetwattana T, Chewaskulyong B, Lee $\mathrm{KH}$, et al. Osimertinib in untreated EGFR-mutated advanced non-small-cell lung cancer. N Engl J Med 2018;378:113-25.

39. Wu YL, Cheng Y, Zhou X, Lee KH, Nakagawa K, Niho S, et al. Dacomitinib versus gefitinib as first-line treatment for patients with EGFR-mutation-positive non-small-cell lung cancer (ARCHER 1050): a randomised, open-label, phase 3 trial. Lancet Oncol 2017;18:1454-66.

40. Sequist LV, Yang JC, Yamamoto N, O’Byrne K, Hirsh V, Mok T, et al. Phase III study of afatinib or cisplatin plus pemetrexed in patients with metastatic lung adenocarcinoma with EGFR mutations. J Clin Oncol 2013; 31:3327-34.

41. Wu YL, Zhou C, Hu CP, Feng J, Lu S, Huang Y, et al. Afatinib versus cisplatin plus gemcitabine for first-line treatment of Asian patients with advanced non-smallcell lung cancer harbouring EGFR mutations (LUX-Lung 6): an open-label, randomised phase 3 trial. Lancet Oncol 2014;15:213-22.

42. Park K, Tan EH, O'Byrne K, Zhang L, Boyer M, Mok T, et al. Afatinib versus gefitinib as first-line treatment of patients with EGFR mutation-positive nonsmall-cell lung cancer (LUX-Lung 7): a phase 2B, open-label, randomised controlled trial. Lancet Oncol 2016;17:577-89.

43. Mitsudomi T, Morita S, Yatabe Y, Negoro S, Okamoto I, Tsurutani J, et al. Gefitinib versus cisplatin plus docetaxel in patients with non-small-cell lung cancer harbouring mutations of the epidermal growth factor receptor (WJTOG3405): an open label, randomised phase 3 trial. Lancet Oncol 2010; $11: 121-8$.

44. Rosell R, Carcereny E, Gervais R, Vergnenegre A, Massuti B, Felip E, et al. Erlotinib versus standard chemotherapy as first-line treatment for European patients with advanced EGFR mutation-positive non-small-cell lung cancer (EURTAC): a multicentre, open-label, randomised phase 3 trial. Lancet Oncol 2012;13:239-46. 


\section{Clinical Cancer Research}

\section{RELAY Subgroup Analyses by EGFR Ex19del and Ex21L858R Mutations for Ramucirumab Plus Erlotinib in Metastatic Non-Small Cell Lung Cancer}

Kazuhiko Nakagawa, Ernest Nadal, Edward B. Garon, et al.

Clin Cancer Res Published OnlineFirst July 22, 2021. $\begin{array}{ll}\text { Updated version } & \text { Access the most recent version of this article at: } \\ \text { doi:10.1158/1078-0432.CCR-21-0273 }\end{array}$

Supplementary Access the most recent supplemental material at:

Material http://clincancerres.aacrjournals.org/content/suppl/2021/08/19/1078-0432.CCR-21-0273.DC1

E-mail alerts Sign up to receive free email-alerts related to this article or journal.

Reprints and To order reprints of this article or to subscribe to the journal, contact the AACR Publications

Subscriptions Department at pubs@aacr.org.

Permissions To request permission to re-use all or part of this article, use this link

http://clincancerres.aacrjournals.org/content/early/2021/08/27/1078-0432.CCR-21-0273.

Click on "Request Permissions" which will take you to the Copyright Clearance Center's

(CCC)

Rightslink site. 